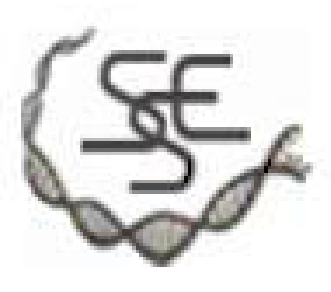

Perspective: Spontaneous Deleterious Mutation

Author(s): Michael Lynch, Jeff Blanchard, David Houle, Travis Kibota, Stewart Schultz,

Larissa Vassilieva, John Willis

Source: Evolution, Vol. 53, No. 3 (Jun., 1999), pp. 645-663

Published by: Society for the Study of Evolution

Stable URL: http://www.jstor.org/stable/2640707

Accessed: 24/07/2010 18:42

Your use of the JSTOR archive indicates your acceptance of JSTOR's Terms and Conditions of Use, available at http://www.jstor.org/page/info/about/policies/terms.jsp. JSTOR's Terms and Conditions of Use provides, in part, that unless you have obtained prior permission, you may not download an entire issue of a journal or multiple copies of articles, and you may use content in the JSTOR archive only for your personal, non-commercial use.

Please contact the publisher regarding any further use of this work. Publisher contact information may be obtained at http://www.jstor.org/action/showPublisher?publisherCode=ssevol.

Each copy of any part of a JSTOR transmission must contain the same copyright notice that appears on the screen or printed page of such transmission.

JSTOR is a not-for-profit service that helps scholars, researchers, and students discover, use, and build upon a wide range of content in a trusted digital archive. We use information technology and tools to increase productivity and facilitate new forms of scholarship. For more information about JSTOR, please contact support@jstor.org.

Society for the Study of Evolution is collaborating with JSTOR to digitize, preserve and extend access to Evolution. 
INTERNATIONAL JOURNAL OF ORGANIC EVOLUTION

PUBLISHED BY

THE SOCIETY FOR THE STUDY OF EVOLUTION

Vol. 53

June 1999

No. 3

Evolution, 53(3), 1999, pp. 645-663

\title{
PERSPECTIVE: SPONTANEOUS DELETERIOUS MUTATION
}

\author{
Michael Lynch, ${ }^{1,2}$ Jeff Blanchard, ${ }^{1}$ David Houle, ${ }^{3}$ Travis Kibota, ${ }^{4}$ Stewart Schultz, ${ }^{5}$ \\ LARISSA VASSILIEVA, ${ }^{1}$ AND JOHN WILlis ${ }^{1}$ \\ ${ }^{1}$ Department of Biology, University of Oregon, Eugene, Oregon 97403 \\ ${ }^{2}$ E-mail: mlynch@oregon.uoregon.edu \\ ${ }^{3}$ Department of Zoology, University of Toronto, Toronto, Ontario M5S 1A1, Canada \\ ${ }^{4}$ Biology Department, Clark College, Vancouver, Washington 98663 \\ ${ }^{5}$ Department of Biology, University of Miami, Coral Gables, Florida 33124
}

\begin{abstract}
Mildly deleterious mutation has been invoked as a leading explanation for a diverse array of observations in evolutionary genetics and molecular evolution and is thought to be a significant risk of extinction for small populations. However, much of the empirical evidence for the deleterious-mutation process derives from studies of Drosophila melanogaster, some of which have been called into question. We review a broad array of data that collectively support the hypothesis that deleterious mutations arise in flies at rate of about one per individual per generation, with the average mutation decreasing fitness by about only $2 \%$ in the heterozygous state. Empirical evidence from microbes, plants, and several other animal species provide further support for the idea that most mutations have only mildly deleterious effects on fitness, and several other species appear to have genomic mutation rates that are of the order of magnitude observed in Drosophila. However, there is mounting evidence that some organisms have genomic deleterious mutation rates that are substantially lower than one per individual per generation. These lower rates may be at least partially reconciled with the Drosophila data by taking into consideration the number of germline cell divisions per generation. To fully resolve the existing controversy over the properties of spontaneous mutations, a number of issues need to be clarified. These include the form of the distribution of mutational effects and the extent to which this is modified by the environmental and genetic background and the contribution of basic biological features such as generation length and genome size to interspecific differences in the genomic mutation rate. Once such information is available, it should be possible to make a refined statement about the long-term impact of mutation on the genetic integrity of human populations subject to relaxed selection resulting from modern medical procedures.
\end{abstract}

Key words.-Deleterious mutation, fitness, mutation, mutation rate, mutational effect.

Received August 21, 1998. Accepted January 25, 1999.

Mildly deleterious mutation has been invoked as a leading explanation for a remarkably diverse array of biological phenomena. For example, in the field of evolutionary genetics, such mutations are thought to be involved in the determination of the magnitude of inbreeding depression (Morton et al. 1956; B. Charlesworth et al. 1990; D. Charlesworth and B. Charlesworth 1987; D. Charlesworth et al. 1992; Deng and Lynch 1996), the maintenance of genetic variation for fitness by selection-mutation balance (Haldane 1937; Kondrashov and Turelli 1992; Houle et al. 1997a; B. Charlesworth and Hughes, in press), the degeneration of $\mathrm{Y}$ chromosomes (B. Charlesworth 1991; Rice 1994), and the evolution of ploidy level (Kondrashov and Crow 1991; Perrot et al. 1991), mating system (Lande and Schemske 1985; Pamilo et al. 1987; Kondrashov 1988; Lynch et al. 1995a), species range (Kawecki et al. 1997), recombination frequency (Charlesworth 1990), and senescence (Hamilton 1966; Partridge and Barton 1993; Charlesworth and Hughes 1996). In the field of molecular evolution, deleterious mutations have been held responsible for the deviations of patterns of variation from the neutral expectation (Kimura 1983; Ohta 1992), the fate of gene duplicates ( $\mathrm{Li}$ 1980; Walsh 1995; Force et al. 1999), the distribution of transposable elements (B. Charlesworth et al. 1992), the maintenance of codon bias by selection-mutation balance (Bulmer 1991; Akashi 1995), and levels of nucleotide diversity in various regions of the genome (Charlesworth et al. 1993, 1995; Hudson and Kaplan 1995; Lynch and Blanchard 1998). The accumulation of mildly deleterious mutations by random genetic drift is also thought to be a significant risk of extinction for small populations of endangered species (Lynch et al. 1993, 1995a,b; Lande 1994) as well as a potential threat to the genetic well-being of our own species (Muller 1950; Crow 1993b, 1997; Kondrashov 1995). This focus on mildly deleterious mutation as a unifying explanation for a broad array of evolutionary phenomena is due in large part to the now classical experiments of 
Mukai and colleagues (Mukai 1964, 1979; Mukai et al. 1972) and subsequent work by Ohnishi $(1977 \mathrm{a}, \mathrm{b}, \mathrm{c})$. Their studies involved specially constructed lines of Drosophila melanogaster that allowed the prolonged propagation of single nonrecombinant chromosomes. Starting with replicate lines containing identical copies of a single ancestral chromosome, after only a few dozen generations the mean viability of the lines had declined substantially and the variance among lines had increased dramatically. Similar results were subsequently obtained by Houle et al. (1992, 1994a), although quantitative comparison with the earlier results was made difficult by the contamination of a control line (Houle et al. 1994b). The observed patterns of line divergence in these experiments are compatible with a diploid genomic deleterious mutation rate on the order of 0.6 per generation or larger and average homozygous effects of mutations on viability on the order of $5 \%$ or less (reviewed in Simmons and Crow 1977; Crow and Simmons 1983; Lynch et al. 1995b). Because viability is only one component of fitness, these results suggest an even higher genomic rate of deleterious mutation for total fitness (Fry et al. 1998), and becaue most mutations have mildly deleterious effects, these are precisely the conditions that can foster the accumulation of a significant mutation load by random genetic drift.

The validity of this classical interpretation of the Drosophila experiments has recently been questioned, with doubts being raised as to whether the genomic deleterious mutation rate is anywhere near as high and the average fitness effects anywhere near as low as suggested. The criticism has come from three directions. First, Keightley $(1994,1996)$ and García-Dorado (1997; García-Dorado and Marín 1998; García-Dorado et al. 1998) have reanalyzed the data of Mukai and Ohnishi, employing two new statistical approaches. The results of these studies conflict with each other, as well as with the classical interpretation, in several respects. Second, two new mutation-accumulation experiments with flies (Fernández and López-Fanjul 1996; Gilligan et al. 1997), which are quite different in design from those of Mukai and Ohnishi, have apparently failed to obtain the magnitude of fitness decline anticipated by the earlier studies, although two additional studies (Shabalina et al. 1997; Fry et al. 1999) obtained results that are in some ways quite compatible with the classical interpretation. Third, recent estimates of the genomic deleterious mutation rate in other organisms (Kibota and Lynch 1996; Keightley and Caballero 1997; Vassilieva and Lynch 1999; Schultz et al., unpubl. ms.) have been lower than those for flies, suggesting that the Drosophila results, even if valid, may not be entirely generalizable to other species.

Given the potentially broad implications of the fitness properties of spontaneous deleterious mutation, our goal is to provide a critical and comprehensive overview of the existing data. We will start by considering some of the more traditional attempts to quantify the mutability of polygenic characters in terms of the variation produced by mutation and then consider the special empirical and analytical approaches employed by Mukai and Ohnishi to elucidate the relative contributions of mutation rates and effects to mutational variance. These overviews will be followed by a summary of more recent empirical results obtained with microcrusta- ceans, nematodes, and plants. We will then consider the distribution-based analytical approaches of Keightley (1994, 1996) and García-Dorado (1997; García-Dorado and Marín 1998; García-Dorado et al. 1998) and the recent Drosophila studies, following this by a broad overview of additional data bearing on the properties of spontaneous mutation. We conclude by arguing that a high (on the order of 0.1 to 1.0 per individual per generation) genomic mutation rate to mildly deleterious mutations is a common feature of multicellular eukaryotes, discussing some empirical approaches that should help clarify our understanding of the fitness consequences of mutations, and considering the potential implications of the existing data for the situation in humans.

Before proceeding, a definition of the types of mutations that we will be considering is in order. A lethal mutation is one that in the homozygous state reduces individual fitness to zero, whereas deleterious mutations have milder effects and neutral mutations have no effect on fitness. Because the distribution of mutational effects is continuous, it is difficult to objectively subdivide the class of deleterious mutations any further, although mutations that reduce fitness to within a few percent of zero are often referred to as sublethals. Our concern will be with the full spectrum of mutations with mild enough effects that there is some chance for their eventual fixation in small populations; effectively, this includes all mutations that reduce fitness by $25 \%$ or less. Deleterious mutations whose selection coefficients are much less than the reciprocal of the effective population size $\left(\ll 1 / N_{e}\right)$ have dynamics that are nearly entirely governed by random genetic drift and various authors refer to them as effectively neutral, whereas mutations with selection coefficients on the order of $1 / N_{e}$ are influenced by the joint forces of selection and drift and are often referred to as nearly neutral or very slightly deleterious. Because effective population sizes differ by many orders of magnitude among species and through time, the bounds on these latter classes of mutations are species specific. Because the absolute fitness effects of effectively neutral and nearly neutral mutations are still at least mildly harmful to their bearers, we regard them as contributors to the deleterious pool, although their contributions may be small depending on their relative incidences.

\section{Mutational Heritability}

The mutational variability of a quantitative trait is expressed by the per generation rate of input of new genetic variance, $\sigma^{2}{ }_{m}$ (Lande 1975; Lynch 1988; Houle et al. 1996), generally referred to as the mutational variance. The most common method of estimation of mutational variance is a divergence experiment in which initially genetically identical replicate lines are propagated from generation to generation through extreme bottlenecks. Such propagation typically involves single full-sibling matings in outcrossing species or single-progeny descent in organisms capable of self-fertilization or clonal reproduction. Through periodic assays of the lines in a common environment, ideally with a parallel control, the rate of increase in the among-line variance can be used to estimate $\sigma^{2}{ }_{m}$. A simple way to see this is to consider a small inbred line with effective size $N$. Letting $U$ be the diploid genomic mutation rate, the expected number of 
TABLE 1. Estimates of the mutational heritability $\left(h_{m}^{2}\right)$ and mutational coefficient of variation $\left(C V_{m}\right)$ for various characters in Drosophila melanogaster. Standard errors of mean estimates are given in parentheses. Where Houle et al. (1996) and Lynch and Walsh (1998) are given as references, there are numerous primary references underlying the pooled estimates.

\begin{tabular}{lccl}
\hline \multicolumn{1}{c}{ Character } & $h_{m}^{2}$ & $C V_{m}$ & \multicolumn{1}{c}{ Reference } \\
\hline Morphological and physiological characters: & & & \\
Abdominal bristle number & 0.0035 & 0.0024 & \multicolumn{1}{c}{ Lynch and Walsh (1998), Houle et al. (1996) } \\
Sternopleural bristle number & 0.0043 & 0.0043 & $\begin{array}{l}\text { Lynch and Walsh (1998), Houle et al. (1996) } \\
\text { Ethanol resistance }\end{array}$ \\
Wing dimensions & 0.0009 & 0.0015 & Weber and Diggins (1990) \\
Body weight & 0.0020 & 0.0014 & Santiago et al. (1992) \\
Thorax length & 0.0047 & 0.0017 & Clark et al. (1995) \\
Enzyme activities & 0.0002 & 0.0005 & Wayne and Mackay (1998) \\
Average & 0.0022 & 0.0005 & Clark et al. (1995) \\
Life-history traits & & $0.0018(0.0005)$ & \\
Longevity & 0.0035 & & \\
Egg-to-adult viability (competitive) & 0.0002 & 0.0195 & Pletcher et al. (1998) \\
Viability (noncompetitive) & 0.0007 & 0.0209 & Fernández and López-Fanjul (1997) \\
Male mating activity & 0.0006 & 0.0065 & Fernández and López-Fanjul (1997) \\
Ovariole number & 0.0005 & 0.0289 & Houle et al. (1994b) \\
Productivity & 0.0022 & 0.0025 & Wayne and Mackay (1998) \\
Fitness & - & 0.0219 & Houle et al. (1994b), Martorell et al. (1998) \\
Average & $0.0013(0.0005)$ & $0.0202(0.0050)$ & Houle et al. (1992) \\
\hline
\end{tabular}

new mutations arising in the line each generation is $U N$. Each new mutation has an initial frequency of $1 /(2 N)$, and for sexually reproducing species, this is also the probability of fixation by random genetic drift provided the deleterious effects are not too great. Letting $2 a$ be the difference between the mean phenotypes of mutant and nonmutant homozygotes, the expected rate of increase of the among-line variance is then equal to $2 U E\left(a^{2}\right)$, which is the product of the expected number of fixations arising between two lines per generation, $2 \times U N \times 1 /(2 N)=U$, and the expected increment of the among-line variance caused by each fixation, $E\left[(2 a-0)^{2}\right] /$ $2=2 E\left(a^{2}\right)$. This result is quite robust in that it does not depend on the degree of dominance of new mutations or on the linkage relationships of loci (Lynch and Hill 1986). Subtle departures from linear divergence can occur in the early generations of a mutation-accumulation experiment if the base genotype is completely lacking in heterozygosity as opposed to being in drift-mutation equilibrium (Lynch and Hill 1986), but the preceding derivation captures the essence of the process.

In the above derivation, we assumed that natural selection is completely ineffective. This ideal situation is not always realized for alleles with effects on fitness. If $s$ is the reduction in relative fitness of a mutant allele with additive effects, the probability of fixation is reduced by a factor of approximately $N s$ when $N s$ is much less than one, and there is virtually no possibility of fixation of mutations for which $N s \gg 1$ (Kimura and Ohta 1971). Thus, the degree of bias in $\sigma^{2}{ }_{m}$ estimates will depend on the joint distribution of effects of mutations on fitness and the trait. Keightley et al. (1993) used data on the fitness effects of P-element insertions influencing bristle traits in Drosophila melanogaster to estimate this joint distribution. From this, they calculated that $\sigma^{2}{ }_{m}$ estimates in full-sibling divergence experiments $(N=2)$ are biased downward by about $30 \%$. This may be an overestimate of the bias for spontaneous mutations, as there is some evidence that $\mathrm{P}$ element insertions have larger effects on fitness than other kinds of spontaneous mutations (Mackay et al. 1992; Keightley 1994). Nevertheless, it should also be noted that lines are commonly lost during mutation-accumulation experiments. To the extent that these losses are the consequence of accumulated mutations, this introduces an additional downward bias to estimates of $\sigma^{2}{ }_{m}$. These considerations suggest that for self-fertilizing and full-sibling mated lines, respectively, alleles that cause a greater than $25 \%$ and $10 \%$ decline in fitness within a culture are highly unlikely to accumulate.

To compare $\sigma^{2}{ }_{m}$ among traits or among species, estimates must be standardized. Two simple nondimensional measures are the mutational heritability $\left(h^{2}{ }_{m}\right)$, which is the ratio of $\sigma^{2}{ }_{m}$ and the environmental variance $\left(\sigma_{E}^{2}\right)$ of the trait, and the mutational coefficient of variation, $C V_{m}=\sigma_{m} / \bar{z}$ where $\bar{z}$ is the trait mean. The mutational heritability is readily estimated from experimental designs such as those discussed above because the within-line component of variance provides an estimate of $\sigma^{2}{ }_{E}$. Because $\sigma^{2}{ }_{m}$ is typically much less than $\sigma_{E}^{2}$, this ratio is essentially equal to the rate of replenishment of the heritability of a character in a genetically depauperate population. The mutational heritability enters a number of formulations in evolutionary theory. For example, for a neutral character with an additive genetic basis, the equilibrium heritability under drift-mutation equilibrium is simply $2 \mathrm{Nh}^{2} \mathrm{~m} l$ $\left(2 \mathrm{Nh}^{2}{ }_{m}+1\right)$. Modifications for this expectation for characters with a nonadditive genetic basis and for asexual organisms are given in Lynch and Hill (1986) and Lynch (1994). The mutational coefficient of variation is correlated with the proportional response to selection resulting from the input of new mutational variance (Houle 1992).

The most recent review of estimates of mutational variance, Houle et al. (1996), considers published results through early 1995. Since then, numerous new estimates have appeared, most in Drosophila melanogaster, and these are compiled with older ones in Table 1. For this species, mutational heritabilities tend to fall in the range $0.0005-0.005$, with only a couple of exceptions. A substantial part of this range may 
be due to sampling error. Some idea of the generality of these results can be acquired by considering the average estimates of $h^{2}{ }_{m}$ (and their approximate standard errors) for other species: life-history characters in the nematode Caenorhabditis elegans, 0.0034 (0.0009) (Keightley and Caballero 1997; Vassilieva and Lynch 1999); life-history characters in the plant Arabidopsis thaliana, 0.0042 (0.0042) (Schultz et al., unpubl. ms.); life-history and growth characters in the microcrustacean Daphnia pulex, 0.0058 (0.0013) (Lynch et al. 1998); morphological, growth-related, and life-history traits in maize Zea mays, 0.0082 (0.0020) (Russell et al. 1963) and in rice Oryza sativa, 0.0028 (0.0003) (Oka et al. 1958; Sakai and Suzuki 1964); and weight and skeletal traits in the mouse Mus musculus, 0.0151 (0.0066) (Bailey 1959; Festing 1973; Keightley 1998). A complete compilation of the existing data is available from the authors. Given the phylogenetic diversity of the organisms for which data are available, and given that selection should cause a downward bias for estimates of $h^{2}{ }_{m}$, we consider it to be an established fact that mutational heritabilities for quantitative characters, including fitness parameters, are usually in the range of 0.001 to 0.01 , although some exceptions probably occur. Below, we will consider in more detail the fact that there are significant differences among species in $h^{2}{ }_{m}$.

Analysis of the existing data, including those shown in Table 1, confirms the patterns identified by Houle et al. (1996). Prior to analysis, we classified traits into five categories based on their presumed relationship to fitness (morphological, size during growth, life history, physiological, and developmental rate/time). Within species, life-history traits and the sizes of body parts during growth tend to have lower $h^{2}{ }_{m}$ than other traits, as can be seen in Table 1 for Drosophila. However, this difference is not statistically significant and could be a simple consequence of the greater downward bias of estimates of $\sigma^{2}{ }_{m}$ for fitness-related traits caused by selection. In contrast, with respect to the mutational $C V$, life-history traits have substantially larger estimates than all other kinds of traits $(P<0.0001)$. The best predictor of $C V_{m}$ in $D$. melanogaster, the species with the largest set of estimates, is an index of the size of the mutational target, the number of loci likely to be involved in the expression of the trait (Houle 1998). Interestingly, the environmental coefficient of variation is highly correlated with $C V_{m}$ (data not shown), suggesting that differences in $h^{2}{ }_{m}$ may be controlled at least as much by $\sigma_{E}^{2}$ as by $\sigma^{2}{ }_{m}$. The correlation between $C V_{m}$ and $C V_{E}$ may be a consequence of the transmission of both genetic and environmental deviations through the same metabolic/developmental pathways.

\section{Bateman-Mukai Estimation of Mutation Parameters}

Mutational variance is a function of the rate at which mutations arise, $U$, and of the average squared effect of a mutant allele, $E\left(a^{2}\right)$. Deciphering the relative contributions of these two subcomponents is a key issue for evolutionary and conservation genetics, as is the estimation of the distribution of mutational effects. For example, the accumulation of deleterious mutations by random genetic drift can be an important risk of extinction for small populations provided there is a common class of mutations with small enough selection co- efficients (Lynch et al. 1993, 1995a,b; Lande 1994). Bateman (1959) had the key insight into how joint estimates of the genomic mutation rate and average effect can be acquired with results from divergence experiments, and his ideas provided the basis for the subsequent analyses of Mukai and Ohnishi. The logic underlying the Bateman-Mukai technique is elegantly simple, requiring only that the mutational rate of change of the mean of a character be known along with the rate of change of the among-line variance.

Consider again a small inbred line with effective size $N$ and diploid genomic mutation rate $U$. The expected number of new mutations arising in the line each generation is again $U N$, with each new mutation having an initial frequency of $1 /(2 N)$. Letting $E(2 a)$ be the difference between the mean phenotypes of mutant and nonmutant homozygotes, the expected rate of change of the mean phenotype caused by the fixation of effectively neutral mutations is $U N \times 1 /(2 N) \times$ $E(2 a)=U E(a)$. Denoting the rates of change in the mean and the variance as $\Delta M$ and $\Delta V$ and recalling the results given above for the rate of change in the among-line variance, we obtain the general relationships:

$$
\begin{aligned}
& U_{\text {min }}=\frac{2(\Delta M)^{2}}{\Delta V}=U\left(\frac{1}{1+C}\right), \\
& \bar{a}_{\text {max }}=\frac{\Delta V}{2 \Delta M}=E(a)(1+C),
\end{aligned}
$$

where $C$ is the squared coefficient of variation of mutational effects, that is, the ratio of the variance of mutational effects, $\sigma^{2}$, and the squared mean effect, $[E(a)]^{2}$. Modifications of these formulae for asexual organisms are given in Lynch (1994).

Because $C$ must be positive, $2(\Delta M)^{2} / \Delta V$ provides a downwardly biased estimate of the genomic mutation rate, usually denoted as $U_{m i n}$, whereas $\Delta V /(2 \Delta M)$ provides an upwardly biased estimate of the average mutational effect, usually denoted as $\bar{a}_{\text {max }}$ (twice this value yielding the homozygous effect). These estimators are biased in the sense that regardless of the sample size, $E\left(U_{\min }\right)<U$ and $E\left(\bar{a}_{\max }\right)<E(a)$. It should be noted, however, that sampling error will cause estimates of $U_{\min }$ to deviate from the true parametric value $U /(1+C)$, and similarly estimates of $\bar{a}_{\max }$ will deviate from $E(a) \times(1$ $+C$ ). Therefore, although the estimators $U_{\min }$ and $\bar{a}_{\text {max }}$ are often referred to as upper and lower bounds on $U$ and $E(a)$, this is not strictly accurate.

The utility of equations $(1 \mathrm{a}, \mathrm{b})$ is that they apply to any character regardless of the form of the distribution of mutational effects, requiring only that there be a directional response of the mean of the character to mutation. Standard errors of the estimates of $U_{\min }$ and $\bar{a}_{\text {max }}$ can be obtained by the delta method (Lynch and Walsh 1998) or by bootstrapping over lines. However, it should also be noted that, due to the fact that the positions of $\Delta M$ and $\Delta V$ are shifted between numerators and denominators in equations $(1 \mathrm{a}, \mathrm{b})$, there is a strong negative sampling covariance between estimates of $U_{\min }$ and $\bar{a}_{\max }$-if one is overestimated, there is a high likelihood that the other will be underestimated.

Mukai and Ohnishi used these estimators in their studies of egg-to-adult viability with lines of Drosophila melanogaster in which mutations were accumulated on nonrecom- 
binant second chromosomes, using a balancer chromosome design developed by Wallace (1956). Under this design, multiple copies of a single wild-type chromosome are initially isolated into different lines. Propagation through males protects the wild-type chromosome from recombination, which does not normally occur in male flies. Heterozygosity of the wild-type chromosome is insured by backcrossing to a control stock carrying two chromosomes, each with a dominant morphological mutation (usually curly wing, $C y$, and plum eye, $\mathrm{Pm}$ ) and a recessive lethal. Throughout the course of the experiment, the $C y / P m$ stock is kept in a state of permanent chromosomal heterozygosity because the $C y$ chromosome is a balancer, which carries multiple inversions that effectively prevent recombination. Each generation, a single male (carrying a wild-type and a marker chromosome) from each mutation-accumulation line is backcrossed to the control line. Because of the recessive lethals carried on the marker chromosomes, all of the singly marked progeny from such crosses must contain the wild-type chromosome from the mutationaccumulation line. Periodically, the relative fitnesses of the mutant chromosomes are evaluated by performing full-sibling matings within the lines, with the ratio of wild-type to marker progeny providing an estimate of relative fitness for the mutant chromosome. For example, in the cross $C y /+\times$ $C y /+$, the expected Mendelian ratio is two $C y$ individuals to one wild-type homozygote, because the $C y$ homozygotes die via the exposure of recessive lethals. Deviations from this ratio arise from differences in the viability of the two genotypes. During the process of mutation accumulation, the relative viability of the wild-type homozygote is expected to decline, assuming that the new mutations are not completely dominant in their effects.

Extrapolating to the entire genome, the investigations of Mukai and Ohnishi yielded average values of $U_{\min }$ equal to approximately 0.6 and average values of $2(1-h) \bar{a}_{\text {max }}$ equal to approximately -0.03 for egg-to-adult viability, where $2 \bar{a}_{\text {max }}$ and $2 h \bar{a}_{\text {max }}$ are the upwardly biased estimates of mean homozygous and heterozygous mutational effects. Because we expect $h<0.5<(1-h)$, these results suggest that the average mutation reduces viability by less than $6 \%$ in the homozygous state and by less than $3 \%$ in the heterozygous state. Similar results were obtained for development time, probably because of the strong pleiotropic effects underlying this character and viability (Mukai and Yamazaki 1971). These estimates are exclusive of chromosomes with mutations having lethal effects. A potential concern is the possibility that the mutation rate of Drosophila males is greater than that of females (Crow 1993a), but a recent analysis (Bauer and Aquadro 1997) suggests that the difference in mutation rate between the sexes in Drosophila may be negligible, so we will adhere to the estimate of $U_{\text {min }} \simeq 0.6$.

There are at least two central concerns with the empirical techniques employed by Mukai and Ohnishi. First, the Wallace technique fails to account for any maternal genetic factors that influence progeny performance because wild-type and marker-bearing individuals have the same mother (a chromosomal heterozygote). Thus, if mutations to genes for maternal transcripts are relatively common, as suggested by recent results of Fry et al. (1998), the preceding estimates of $U$ may be biased downward (beyond the statistical bias noted above). Second, because the $C y / P m$ stock was maintained as a large population throughout the course of the experiments, the introduction of rare beneficial alleles by mutation may have resulted in adaptive evolution by the balancer chromosomes. By inflating the deviation between the performance of mutation-accumulation and control lines, this would presumably cause an overestimate of the actual rate of fitness decline in the mutation-accumulation lines. However, the opposite could also occur if the adaptation to the control environment had negative pleiotropic effects on fitness in the assay environment.

Fry et al. (1999) recently repeated the work of Mukai and Ohnishi, using an essentially identical balancer-chromosome approach. The rate of decline in mean viability in this study was not significantly different than the average value from the Mukai and Ohnishi studies, but the rate of increase in the among-line variance was about four times higher. This resulted in Bateman-Mukai estimates of the genomic deleterious mutation rate of approximately 0.1 and of the quantity $2(1-h) \bar{a}_{\max }$ of approximately -0.11 . It does not appear that the deviation of these new estimates from the older ones is a simple consequence of sampling error. A significant question that remains to be answered is whether the differences reflect strain-specific variation in mutational properties. Given that transposition is a major source of mutation in flies and that the genomic transposition rate can vary substantially among lines (Nuzhdin et al. 1996; Pasyukova et al. 1998), such variation seems very likely.

The Bateman-Mukai method of analysis has been applied to three other eukaryotic systems: a 30-generation mutationaccumulation experiment involving clones of the cladoceran Daphnia pulex (Lynch et al. 1998), a 10-generation experiment involving self-fertilizing lines of the plant Arabidopsis thaliana (Schultz et al., in prep.), and two independent 50and 60- generation experiment involving self-fertilizing lines of the nematode Caenorhabditis elegans (Keightley and Caballero 1997; Vassilieva and Lynch 1999). In all of these studies, mutations were accumulated throughout the entire genome and a control was maintained in an inert evolutionary state prior to its contemporaneous assay with the mutationaccumulation lines-stored as diapausing embryos of an obligately asexual strain in the case of Daphnia, as seed in the case of Arabidopsis, and as frozen larvae in the case of Caenorhabditis. Estimates of $U_{\min }$ for fitness-related traits in Daphnia average to 0.76 (0.33), which is quite compatible with the Mukai and Ohnishi results for viability in D. melanogaster (Table 2). However, those for C. elegans and A. thaliana are considerably lower, averaging $0.05(0.02)$ and $0.04(0.02)$, respectively (Table 2 ).

\section{ReAPPRAISAls of Old Data}

There have been several recent attempts to reevaluate the analyses of Mukai and Ohnishi. Rather than simply using information on the change in the mean and variance of mutant lines to obtain biased estimates of the mutation parameters, these new analyses attempt to extract unbiased estimates from the observed form of the distribution of line means, in effect by relying on the information content in the higher moments (skewness, kurtosis, etc.) of the distribution of line means. 
TABLE 2. Downwardly biased estimates of the genomic mutation rate $\left(U_{m i n}\right)$ and upwardly biased estimates of the average mutational effect relative to the mean phenotype $\left(\bar{a}_{\max }\right)$.

\begin{tabular}{|c|c|c|c|}
\hline Character & $U_{\min }$ & $\bar{a}_{\max }$ & Reference \\
\hline \multicolumn{4}{|l|}{ Daphnia pulex: } \\
\hline Size at birth & $1.513(1.345)$ & $-0.005(0.005)$ & \multirow[t]{5}{*}{ Lynch et al. (1998) } \\
\hline Size at maturity & $0.729(0.627)$ & $-0.008(0.004)$ & \\
\hline Adult instar duration & $0.046(0.007)$ & $-0.010(0.001)$ & \\
\hline Clutch size & $0.201(0.103)$ & $0.039(0.011)$ & \\
\hline Survival to maturity & $1.946(0.854)$ & $-0.008(0.003)$ & \\
\hline \multicolumn{4}{|l|}{ Caenorhabditis elegans: } \\
\hline Productivity & $0.023(0.042)$ & $0.120(0.114)$ & \multirow{3}{*}{ Vassilieva and Lynch (1999) } \\
\hline Survival to maturity & $0.012(0.007)$ & $-0.068(0.027)$ & \\
\hline Longevity & $0.129(0.089)$ & $-0.024(0.010)$ & \\
\hline \multicolumn{4}{|l|}{ Arabidopsis thaliana: } \\
\hline Germination rate & $0.002(0.642)$ & $-0.625(2.464)$ & \multirow[t]{2}{*}{ Schultz et al. (in prep.) } \\
\hline Fruit set & $0.003(0.392)$ & $-0.211(5.315)$ & \\
\hline
\end{tabular}

In the first of such studies, Keightley (1994) performed a maximum-likelihood (hereafter, ML) analysis, assuming a Poisson distribution of mutation numbers and a gamma distribution of mutational effects. His analyses were restricted to the final assays of the mutation-accumulation lines of $\mathrm{Mu}$ kai et al. (1972) and Ohnishi (1974), and in the absence of contemporaneous controls for the assay generations, the control means and variances were inferred from other data and assumed to be known without error. Extrapolating from the results for the second chromosome to the entire genome, Keightley's (1994) results imply a minimum average estimate of $U$ of approximately 0.52 , which is very similar to the Bateman-Mukai estimate. Conditional on these estimates, the mean difference between the effects of homozygous and heterozygous mutations on viability was estimated to be on the order of 0.5 to $1.0 \%$ (slightly less than the $3 \%$ that we cited above), and the distribution of mutational effects was inferred to be highly leptokurtic (L-shaped). However, the actual likelihood surface suggested an even more extreme situationa mutation rate approaching infinity and an average mutational effect approaching zero. Similar asymptotic behavior of ML estimates was noted recently for eight of nine characters in an analysis of Drosophila lines mutagenized by EMS (Keightley and Ohnishi 1998) as well as in the study of Fry et al. (1999).

Keightley (1994) chose to use a gamma distribution of mutational effects because of the broad array of forms that it can assume, from L-shaped to approximately normal. However, one potential limitation of the gamma distribution is its unimodality. In a more recent analysis, Keightley (1996) attempted to account for this by using a model in which a fraction of the mutations was assumed to be gamma distributed with the remaining fraction being of constant effect. The actual parameter estimates are not given in this second paper, but the ML estimates of $U$ again apparently converged on infinity, with the minimum estimates compatible with the data averaging to 0.60 , consistent with the classical interpretation of the data. A mixed distribution, consisting of a highly Lshaped gamma distribution and a constant effect of small value, was found to fit the data better than the gamma distribution alone. Keightley (1996) also raised the possibility that the change in mean fitness in the mutation-accumulation lines may have been an artifact of adaptive evolution in the balancer chromosomes rather than a consequence of mutational degradation of the experimental chromosomes.

In a third reevaluation of the results of Mukai and Ohnishi, García-Dorado (1997) employed minimum-distance (hereafter, MD) analysis to estimate the parameters of the mutation process that are most consistent with the final distribution of line means, conditional on the observed estimate of the rate of increase in among-line variance. Like Keightley (1994, 1996), she assumed a Poisson distribution of mutation numbers and an extrapolated control mean and variance. However, the distributions of mutational effects explored by GarcíaDorado (1997) were more general (asymmetrical reflected gammas, and mixtures of gamma and normal distributions). Contrary to Keightley (1994, 1996), García-Dorado (1997) concluded that the genomic deleterious mutation rate is on the order of only 0.05 per individual per generation, and a more recent set of analyses led to even lower estimates ( $U$ $\simeq 0.01$ ) (García-Dorado et al. 1998). Both analyses inferred a unimodal distribution of deleterious viability effects with a very high mean (on the order of 6 to $23 \%$ ), with mutations having effects near zero being very rare and those with effects in excess of $15 \%$, and even as high as $40 \%$, being relatively common. For the dataset of Mukai et al. (1972), GarcíaDorado inferred a beneficial mutation rate of $12 \%$, and argued that her statistical fits to the data are uniformly better than those of Keightley (1994).

These new types of analyses provide a novel framework for characterizing the properties of polygenic mutations. However, there are a number of unresolved issues concerning their use. First, as noted above, distribution-based methods are highly dependent on observed levels of skewness and kurtosis. Since estimates of higher-order moments are notoriously unreliable unless sample sizes are enormous, this greatly limits the statistical power of distribution-based methods (see Keightley and Ohnishi 1998; Keightley 1998). 
García-Dorado (1997) was able to recover unbiased estimates of the mutation parameters from simulated data, but her simulations employed the same distributions that were assumed by the model. To what extent do violations of the assumptions about the forms of the distributions underlying the data influence the parameter estimates derived by ML and MD? The fact that García-Dorado and Keightley reached such different conclusions from the same data clearly indicates that this is not a trivial issue. In addition to making various assumptions about the distribution of mutational effects, both Keightley (1994, 1996) and García-Dorado (1997) assumed that the sampling errors associated with line means are normally distributed. Deviations from this latter assumption, which clearly occur (Keightley and Ohnishi 1998), are of special concern because the essence of distribution-based techniques is to infer the properties of mutations from the departure of the distribution of the mutation-accumulation lines from the assumed normal distribution of the control.

Second, the fact that García-Dorado (1997) and GarcíaDorado et al. (1998) obtained better fits to the data than did Keightley (1994) is not surprising, because their analyses typically incorporated more parameters. More complex model almost always yield better fits and should not be taken as a priori evidence for greater biological plausibility. Although not impossible, the high densities of deleterious mutations with large homozygous effects (substantially in excess of $15 \%$ ) posited by García-Dorado (1997) and García-Dorado et al. (1998) seem to be incompatible with what can be achieved in mutation-accumulation experiments unless they are highly recessive and sheltered from selection by a Plum chromosome.

Third, most of the analyses favored by García-Dorado and colleagues ignore the observed change in mean fitness in the mutation-accumulation lines entirely. Instead, under the assumption that this distribution was originally normal, they estimate the mutation parameters solely from the distribution of line means at the end of the mutation-accumulation experiment, with no reference to a control. With this sort of approach, it does not seem surprising that an extremely low mutation rate to very deleterious alleles is estimated because the observed distributions of fitness typically have long tails to the left, but are otherwise approximately normal. When García-Dorado and colleagues performed analyses conditional on the observed changes in both the mean and the variance, the results were quite compatible with the classical interpretation of Mukai and Ohnishi's results, and thus were in approximate agreement with Keightley's (1994) first study. García-Dorado (1997) favors analyses based only on the variance, arguing again that the decline in mean fitness in themutation-accumulation lines is an artifact of adaptive evolution in the control.

To our knowledge, there is no direct observational support for or against a change in the mean fitness of the marker chromosomes in any of the experiments of Mukai and Ohnishi, but the fact that fairly consistent results were obtained in several independent experiments seems to argue against such problems, as this would require the controls of different experiments to evolve in the same manner. Moreover, in their balancer chromosome work, Fry et al. (1999) obtained compelling evidence for an absence of control line evolution.
Nevertheless, if the control chromosomes in the Mukai and Ohnishi experiments did actually evolve, then it is not clear that the data are reliable for any sort of analysis, including methods based solely on the among-line variance. The reason for this is that evolution of the balancer chromosomes would not only influence the rate of change in mean fitness, but also the rate of change in the higher-order moments, a point that seems not to have been taken into consideration in the studies described above.

\section{Mutational Degradation in Fly Populations}

To sum up to this point, when extrapolated over the entire genome, all of the results from balancer-chromosome experiments suggest that if released from selection, spontaneous deleterious mutations in Drosophila would cause a 1-2\% loss in viability per generation. Recent studies with Drosophila have further evaluated this idea, but with rather different results. Unlike the experiments of Mukai and Ohnishi, these newer studies have allowed mutations to accumulate throughout the entire genome, without the use of balancer chromosomes. A brief review of these studies has been given by Keightley et al. (1998).

Rather than repeatedly bottlenecking lines, Shabalina et al. (1997) encouraged the accumulation of mutations by maintaining a large population in way that minimized the possibility of among-family selection. Starting with a population of flies from the wild, 100 randomly mated pairs were established. Their progeny were allowed to develop under low density, noncompetitive conditions, and then from each sibship single males and females were extracted and randomly mated across sibships to produce the next generation. Such propagation was continued for a total of 30 generations. With this "middle-class neighborhood" design, every mating pair contributes equally to the next generation. Two controls were maintained in this study, one kept in a cyropreserved state and the other kept at a low enough temperature that only three generations had been passed by the time of analysis of the mutation-accumulation experiment. Relative to the control values, when assayed under low density conditions, eggto-imago survivorship declined by approximately $0.2 \%$ per generation, motility by about $0.1 \%$ per generation, and fecundity by about $0.003 \%$ per generation. Although small, all of these changes were statistically significant. In contrast, under competitive conditions, the relative performance of the middle-class neighborhood individuals declined by $2.2 \%$ per generation, in striking agreement with the results of Mukai and Ohnishi.

A second middle-class neighborhood experiment (Gilligan et al. 1997), appears to have yielded very different results than that of Shabalina et al. (1997). However, there are numerous aspects of the results of this study that are difficult to explain, including the fact that mean fitness tended to increase at lower population sizes. We will not discuss this experiment any further because there may have been substantial evolution in the reference population (R. Frankham, pers. comm.).

Shabalina et al. (1997) argue that the advantage of the middle-class neighborhood design is its reliance on individuals that have not been genetically altered by inbreeding or 
by nonrecombinant chromosome propagation through crosses to a balancer stock. This is an appropriate goal in that the purpose of most mutation-accumulation studies is to evaluate the consequences of mutations on genetic backgrounds with normal fitness and normal levels of recombination and outcrossing. However, the utilization of a genetically variable base population extracted from the wild also introduces a number of potential problems.

First, the use of a base population harboring significant genetic variance for fitness can partially obscure the pattern of buildup of genetic load due to new deleterious mutations. Until all base-population alleles have either been lost or fixed by random genetic drift, there will be some contribution to the change in mean fitness from inbreeding depression resulting from the exposure of preexisting recessive mutations. Roughly speaking, this requires at least $4 N_{e}$ generations. Under the middle-class neighborhood design, $N_{e}$ is twice the number of mating individuals, so for an experiment of the size employed by Shabalina et al. (1997), 100 pairs with equal family sizes (implying $N_{e} \simeq 400$ ), approximately 1600 generations would need to expire before the evolutionary dynamics were solely a consequence of new mutations. Shabalina et al. (1997) reason that if the middle-class neighborhood is kept at a sufficiently large size, then inbreeding can be largely avoided in the early stages of mutation accumulation (in their study, the average inbreeding coefficient only rose to $4 \%$ ). In principle, rather than relying on a very large randomly mating population as Shabalina et al. did, inbreeding can be avoided entirely by use of a circular system of mating or a maximum-avoidance of inbreeding scheme (Kimura and Crow 1963). Under the latter scheme, for example, if $N=2^{m}$ is the size of the population, then no inbreeding occurs until generation $m+2$.

A second, less tractable issue is the possibility that recombinational breakup of epistatic combinations of genes may lead to a change in mean fitness without any changes in allele frequencies by random genetic drift. A series of investigations of the fitness properties of intact versus recombinant chromosomes in species of Drosophila revealed an average $10 \%$ decline in fitness per chromosome associated with a single round of recombination (Spassky et al. 1958; Dobzhansky et al. 1959; Spiess 1959; Krimbas 1961; Spiess and Allen 1961), so we do know that such changes can occur. The recombinational breakdown observed in these Drosophila experiments may be extreme in that the assays involved chromosomal homozygotes, but there are less contrived examples of changes in mean phenotypes in response to recombination in Daphnia (Lynch and Deng 1994; Deng and Lynch 1997). Given the small degree of change that is typically measured in a mutation-accumulation experiment, it would not take much recombinational breakdown to greatly bias the results of a middle-class neighborhood experiment initiated from a wild population, so there is a clear need for more work on this problem. One approach might be to initiate a middle-class neighborhood experiment with a cross between two distant populations (to maximize the initial disequilibria). If the apparent rate of mutational decay remained the same, this would weaken the argument that recombinational breakdown has biased the results.

Third, whereas the middle-class neighborhood design should minimize selection among families, it does not eliminate within-family selection. Of course, conventional mutation-accumulation experiments using bottlenecked lines do not eliminate within-line selection either, but here the opportunity for adaptive evolution is reduced because of the lower level of within-line genetic variance (derived entirely from mutation) and the higher magnitude of drift in inbred lines. Substantial evidence exists for the rapid evolution of competitive ability in newly collected populations of Drosophila raised in a novel laboratory environment (Ayala 1968; Frankham and Loebel 1992; Shabalina et al. 1997), even in those of small size (Latter and Mulley 1995), so it seems likely that selection in a middle-class neighborhood setting will promote to some degree the base population alleles that are most advantageous in the laboratory environment. This would partially erase the effects of the mutationaccumulation process, leading to conservative estimates of mutation pressure. Shabalina et al. (1997) attempted to minimize within-family selection by carefully optimizing rearing conditions, but approximately $20 \%$ of eggs laid still did not survive to adulthood during the mutation-accumulation phase.

A fourth concern with studies of this sort is the evolutionary stability of the controls. Over the course of the Shabalina et al. (1997) experiment, the recovery rate of frozen embryos declined from $18 \%$ to $8 \%$ and the possibility that surviving embryos have higher than average fitness has not been ruled out. If selective mortality of control individuals does occur, then estimates of the mutation load in the experimental lines would be inflated. However, if cryopreservation induces mutations with small deleterious effects or if cold controls experience some mutational deterioration, this would tend to reduce the estimates of mutation load in the experimental lines. Using an assay for X-linked recessive lethals, Houle et al. (1997b) found no significant increase in the mutation rate in frozen embryos, but future work is needed to evaluate whether these results can be extended to mutations with small effects.

In spite of these limitations, we regard the middle-class neighborhood design as a promising innovation for the estimation of recurrent mutation pressure, particularly if withinline mortality and infertility can be kept to a low level and if the problem of recombinational breakdown can be definitively ruled out. A particularly attractive feature of this design is that it opens up the opportunity for studying the properties of polygenic mutation in organisms that cannot be propagated easily as clones or as inbred lines. However, a serious limitation of the middle-class neighborhood design is its probable restriction to the measurement of the rate of change in mean fitness. It seems very unlikely that accurate estimates of mutational variance can be acquired in a system that harbors substantial genetic variance at the outset.

Finally, we note that Keightley (1996), Keightley and Ohnishi (1998), García-Dorado (1997), and García-Dorado et al. (1998) have called attention to a recent mutation-accumulation experiment with 200 full-sibling mated lines of Drosophila (Fernández and López-Fanjul 1996), arguing that it provides further evidence for a low rate of fitness decline by mutation accumulation. Unlike the experiment of Shabalina et al. (1997), this experiment was initiated with a highly 
inbred stock, so the changes in the experimental lines (over a period of approximately 100 generations) are unlikely to be a consequence of alleles segregating in the base population or of recombinational breakdown. However, some aspects of the Fernández and López-Fanjul (1996) study raise questions as to whether the results are strictly comparable with those of Mukai and Ohnishi.

First, the analysis of line means in Fernández and LópezFanjul (1996) was not accompanied by a contemporaneous analysis of the control. Although a control population was maintained in a circular mating scheme involving approximately 800 individuals throughout the course of the experiment, it was only compared against a few (two to four in each direction) of the most extreme mutation-accumulation lines. For pupa-to-adult viability, the high lines exhibited an average $3 \%$ decline in performance, whereas the low lines exhibited an average 16\% decline. For egg-to-pupa viability, the high lines exhibited an average $3 \%$ increase (not significant) in performance, whereas the low lines exhibited an average $16 \%$ decline. For egg production, the high lines exhibited an average $75 \%$ increase in performance, while the low lines exhibited an average $22 \%$ increase. These limited results suggest that the mutation-accumulation lines in this study evolved substantially, although the numbers cited may be biased as a result of the evolution of the control population. With approximately 800 individuals incurring 100 generations of mutations, evolution of the control seems likely in this study.

Second, the opportunity for selection over the course of an experiment as long as that of Fernández and López-Fanjul may have reduced the rate of accumulation of deleterious mutations. In an experiment with a very similar design, but with average line sizes of approximately 10 individuals per generation, Mackay et al. (1995) found that selection against extreme phenotypes causes a dramatic decline in the rate of line divergence of bristle numbers over time. Presumably, the efficiency of selection was lower in the lines of Fernández and López-Fanjul (1996) because they were based on fullsibling mating. However, as in most studies of this sort, the authors relied on backup individuals to guard against line extinction. The frequency with which backups were relied upon was not reported, but $12 \%$ of the lines were lost over the course of the experiment, so the number could not have been trivial. One indirect line of evidence that selection may have reduced the rate of mutation accumulation (acknowledged by the authors) is the fact that the average mutational heritability estimated from the rate of line divergence in this study, 0.0007 (0.0001), is about five times lower than that observed in other studies on life-history traits in Drosophila (Table 1).

Finally, the assays performed by Fernández and LópezFanjul (1996) involved much more benign conditions than the competitive environments of the Mukai and Ohnishi experiments. As noted below, there is now substantial evidence that the expressed mutation load is magnified in harsh environments.

\section{Additional Evidence for High Genomic Mutation RATES FOR FITNESS}

Resolution of the issue of whether the genomic deleterious mutation rate is as small as 0.01 or as high as 1.0 per indi- vidual per generation is critical to the fields of evolutionary and conservation genetics because most of the theoretical studies in these areas have assumed $U \simeq 1.0$. If the genomic deleterious mutation rate is typically 100 times lower than assumed, then many of the evolutionary phenomena outlined in the introduction must be due to other causes. Here, we consider a number of features of Drosophila melanogaster that provide further support for the opinion that genomic mutation rates for fitness-related traits in this species are more on the order of 1.0 than 0.01 .

First, substantial evidence (summarized in table 10.2 of Lynch and Walsh 1998) indicates that the average inbreeding depression for egg-to-adult viability in D. melanogaster is equivalent to a $44 \%$ reduction under complete homozygosity. Moreover, studies with three species of Drosophila have shown that the effective number of lethals on average second and third chromosomes is in the range of 0.5 to 0.8 (table 10.5 of Lynch and Walsh 1998). Because each haploid chromosome constitutes about $40 \%$ of the haploid genome, this suggests that the average drosophilid gamete carries approximately 1.6 lethal equivalents, which is about the same as observed in many vertebrates (table 10.4 of Lynch and Walsh 1998). Inbreeding loads of this magnitude are not easily explained with a genomic deleterious mutation rate on the order of 0.01 . Under mutation-selection balance, the equilibrium effective number of lethals per gamete is approximately $U(1$ $-\tilde{h}) / 2 \tilde{h}$, where $\tilde{h}$ is the harmonic mean dominance coefficient, so if $U=0.01$, then $\tilde{h}$ must equal 0.003 . In other words, to account for the observed inbreeding load with $U=0.01$, most deleterious mutations must be nearly completely recessive, a situation that is clearly inconsistent with the data (Simmons and Crow 1977; Crow and Simmons 1983; Charlesworth and Charlesworth 1987; Houle et al. 1997a; Latter 1998; Lynch and Walsh 1998). Increasing the genomic mutation rate 10 -fold to 0.1 still requires an unrealistically low average dominance coefficient $(0.03)$. If, however, $U_{\min }$ $=1.00$, then $\tilde{h} \geq 0.19$, which is much more compatible with existing data.

A number of authors have also called attention to the fact that standing levels of genetic variance for fitness in Drosophila populations are roughly compatible with the classical interpretation of the results of Mukai and Ohnishi under the assumptions of mutation-selection balance (Mukai and Yamaguchi 1974; Keightley 1994; Charlesworth and Hughes, in press), although $U \simeq 1.0$ does not appear to be entirely adequate. Thus, one of the greatest challenges to the hypothesis that $U \simeq 0.01$ in flies is the need to provide alternative explanations for the properties of natural populations. In principle, inbreeding depression and genetic variation may be maintained by some sort of balancing selection (such as frequency-dependent or temporally fluctuating selection or functional overdominance), rather than selection-mutation balance, but we know of no compelling evidence that this is the case for viability in Drosophila.

Second, per character mutation rates of 0.1 or greater are consistent with what we know about the rates of various types of mutation in D. melanogaster. For example, it is well established that the mutation rate to lethals in $D$. melanogaster is on the order of 0.02 to 0.05 per zygote (Crow and Simmons 1983; Fry et al. 1999). Given that most genomic changes 
(small insertions/deletions and nucleotide substitutions) are unlikely to be lethal, it seems virtually certain that the rate of origin of mildly deleterious mutations must exceed the lethal rate.

A survey of 18 transposable element families in $D$. $m e$ lanogaster suggests that the average number of transpositions per zygote is on the order of 0.8 per generation (Nuzhdin and Mackay 1995; Lyman et al. 1996). In addition, the rate of nucleotide substitutional mutation in Drosophila has been estimated to be about $1.6 \times 10^{-8}$ per site per year (Sharp and Li 1989). Assuming flies go through approximately five generations each year, and noting that the Drosophila genome contains approximately $4 \times 10^{8}$ bases, these results imply that the average number of nucleotide substitutional mutations per genome is at least 1.3 per generation. Summing these two types of mutation and noting that we have ignored other types (e.g., those resulting from small nucleotide insertions/deletions, unequal crossing over, and so on), it is clear that the total genomic mutation rate in $D$. melanogaster must exceed 2.1 per generation. A large fraction of these mutations may have negligible effects on fitness. Nevertheless, supposing the total genomic mutation rate for flies is 3.0 , then unless $<3 \%$ of all new mutations influence fitness, these results provide independent evidence that the genomic deleterious mutation rate for this species is in excess of 0.1 .

Third, analyses of transposable-element induced mutations for bristle numbers provide additional indirect evidence for a relatively high genomic deleterious mutation rate. Focusing on the effects of single P-element insertions on abdominal and sternopleural bristle number in D. melanogaster, Lyman et al. (1996) estimated the average scaled mutational effects, $E\left(a^{2}\right) / \sigma_{E}^{2}$, for these two traits to be equal to 0.070 and 0.205 , respectively. A substantial number of studies on these traits yield average estimates of the mutational heritabilities of 0.0035 for abdominal bristle number and 0.0043 for sternopleural bristle number (Table 1). Noting that the mutational heritability of a trait is equal to $U E\left(a^{2}\right) / \sigma_{E}^{2}$, these results imply genomic mutation rates for abdominal and sternopleural bristle numbers of approximately 0.05 and 0.02 , respectively. Similar calculations applied to the results of Clark et al. (1995) yield even higher estimates of $U$ for body weight (0.29) and enzyme activities (0.14). These calculations assume that mutational effects associated with P-element insertions are reasonably representative of the effects of the full spectrum of mutations. If the effects of P-element insertions are unusually high, then these indirect estimates of $U$ would need to be upwardly adjusted. There is substantial evidence that most bristle-number mutations have negative pleiotropic effects on fitness (Mackay et al. 1992, 1994, 1995; Fry et al. 1995; Nuzhdin et al. 1995), so given that many additional characters must influence fitness, then these results for morphological and metabolic characters provide further evidence that the genomic deleterious mutation rate in this organism is well in excess of 0.1 .

To this point, we have primarily focused on data from Drosophila melanogaster, so it is worth considering whether the high apparent genomic mutation rate for this species is aberrant. Attempts have been made to estimate genomic mutation rates for quantitative traits in a few other species by directly counting major phenotypic differences that have aris- en in mutation-accumulation experiments. In an examination of 27 skeletal traits in inbred strains of laboratory mice, Grewal (1962) obtained an average estimate of 0.022 mutations per character per zygote per generation; in a more extensive study with the same traits, Hoi-Sen (1972) obtained essentially the same result. Studies of a number of reproductive characters in maize using descendants of double-haploids (Sprague et al. 1960) and inbred lines (Russell et al. 1963) yielded average estimates of $U$ equal to 0.090 and 0.056 . All of these estimates are likely to be downwardly biased, perhaps substantially so, because mutations with insufficiently large effects are statistically undetectable, and thus not counted, in these types of studies.

Mutation-accumulation experiments are extremely labor intensive and difficult to apply to many species. An alternative approach to characterizing the features of the deleterious mutation process considers the distribution of fitness in a natural population. Assuming a population in mutationselection balance, Morton et al. (1956) and Mukai and Yamaguchi (1974) first suggested how the genomic deleterious mutation rate in a random-mating population might be estimated from the observed response of mean fitness to inbreeding, and Charlesworth et al. (1990) extended this approach to interpret the consequences of outcrossing in normally self-fertilizing populations. Both techniques require an estimate of the average degree of dominance of mutant alleles. A more general approach, which yields joint estimates of $U$ and the average heterozygous and homozygous effects of mutations, has been developed by Deng and Lynch (1996) and extended by Deng (1998). This method requires estimates of both the mean fitness and the genetic variance for fitness in inbred and outbred generations, but it does not require an estimate of the average degree of dominance. If a substantial fraction of the standing genetic variance is maintained by balancing selection rather that by mutation-selection balance, then these methods will overestimate $U$. In highly selfing populations, functional overdominance for fitness will be ineffective at maintaining genetic variance (Kimura and Ohta 1971) due to the low heterozygosity of individuals, so the application of the Charlesworth et al. (1990) and Deng and Lynch (1996) methods to selfing populations may be quite robust, assuming other forms of balancing selection are not of great importance.

Several estimates of $U$ have been obtained with these techniques. In a survey of the literature on inbreeding depression in obligately self-fertilizing plants, Charlesworth et al. (1990) obtained average estimates of $U$ of at least 0.5 for individual fitness components, Johnston and Schoen (1995) inferred estimates of $U$ for total fitness in populations of the annual plant Amsinckia in the range of 0.2 to 0.9 , and D. Charlesworth et al. (1994) obtained estimates of $U \simeq 0.8$ and 1.5 for two species of Leavenworthia. Applications of the technique of Deng and Lynch (1996) to two populations of Daphnia led to an average estimate of $U$ for clutch size of 0.9 (Deng and Lynch 1997).

\section{Additional Evidence for Small Average Deleterious EfFects of Mutations}

We now consider additional direct lines of evidence that are consistent with the idea that the majority of mutations 


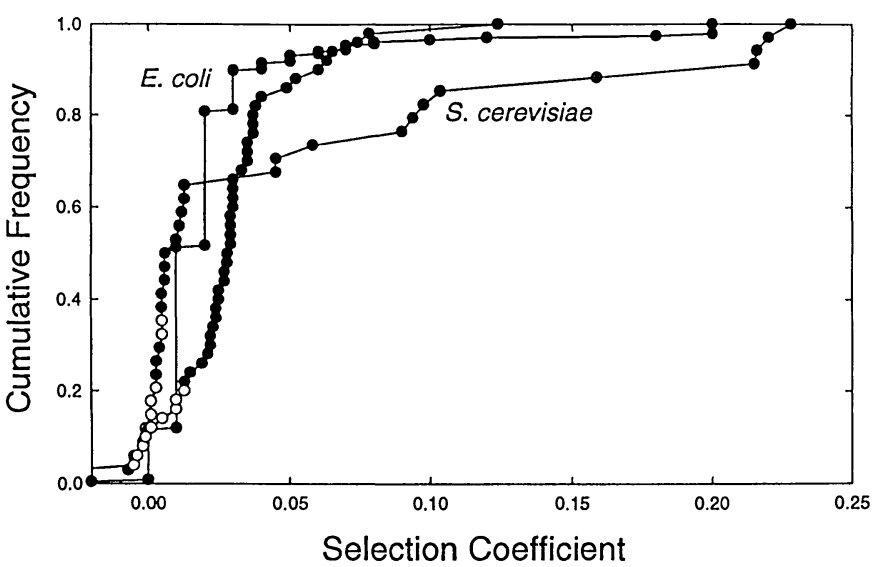

FIG. 1. The cumulative frequency distribution of mutational effects on fitness for a bacterium Escherichia coli (Kibota and Lynch 1996; Elena et al. 1998) and a yeast Saccharomyces cerevisiae (Thatcher et al. 1998). The Elena et al. (1998) and Thatcher et al. (1998) studies evaluated the consequences of single large insertions on the growth potential of mutants relative to control individuals. According to the authors, most of the 226 estimated selection coefficients in the study of Elena et al. (1998) were significant, whereas the few nonsignificant coefficients from the study of 31 insertion lines of Thatcher et al. (1998) are denoted by open circles. The lines of Kibota and Lynch (1996) contain a variable number of spontaneous mutations accumulated over a period of approximately 7000 cell divisions, with the average number being approximately one per line.

have only small (less than 5\%) effects on quantitative characters, including fitness. First, for D. melanogaster, analyses of the effects of P-element insertions on bristle numbers by Lyman et al. (1996) and on metabolic activities by Clark et al. (1995) suggest that mutations with the smallest effects have the highest densities. These results provide direct evidence for the idea, first suggested by Keightley's (1994) ML analysis, that the distribution of mutational effects in this species is highly L-shaped. Even null alleles for allozyme loci in $D$. melanogaster generally do not lead to lethality or sterility in the homozygous state and reduce fitness by only approximately $0.2 \%$ in the heterozygous state (Langley et al. 1981). Estimates of the frequency distribution of mutational effects on fitness in microbes also strongly support the view that most mutations have only mildly deleterious effectseven for large insertions into coding regions, in excess of $70 \%$ of mutations have selection coefficients less than 0.02 (Fig. 1).

Second, Bateman-Mukai estimates of the maximum average mutational effect in organisms other than Drosophila are usually small. For example, a mutation-accumulation experiment with lines of the bacterium $E$. coli yielded an upwardly biased estimate of the average mutational effect on exponential growth rate of $1.2 \%$ (Kibota and Lynch 1996), which is quite consistent with the data in Figure 1. In Daphnia, the average heterozygous effect of a new mutation on life-history characters is no greater than $2.5 \%$ (Table 2). The average (upwardly biased) estimates for life-history characters in the nematode $C$. elegans and the plant $A$. thaliana are higher-on the order of $14 \%$ and $20 \%$ for the mean homozygous effect, respectively (Table 2 ).

As noted above, the degree to which the Bateman-Mukai estimates of $U_{\min }$ and $\bar{a}_{\max }$ are biased is a function of the squared coefficient of variation of mutational effects $(C)$ (eq. $1 \mathrm{a}, \mathrm{b})$. Only limited data are available for the distribution of mutational effects, but all of the existing information suggests a high degree of leptokurtosis, with the form of the distribution being more L-shaped than an exponential distribution. For example, for Drosophila, estimates of $C$ are on the order of 6,24 , and 17 , respectively, for abdominal bristle numbers, sternopleural bristle numbers, and viability (Keightley 1994). For growth rate in $E$. coli, $C \simeq 7$ (Elena et al. 1998), and for competitive ability in $S$. cerevisiae, $C \simeq 2.3$. These observations suggest that the actual average effects of deleterious mutations may be several times lower than the upper-bound estimates cited above (and conversely, that actual genomic deleterious mutation rates may be several times higher than the lower-bound Bateman-Mukai estimates reported in Table 2).

Third, a comparison of standing levels of genetic variance $\left(\sigma^{2}\right)$ with the rate of input of new variance by mutation $\left(\sigma^{2}{ }_{m}\right)$ provides some indirect insight into the average magnitude of selection against heterozygous mutations. Under the assumption that the observed variation is a consequence of mutation-selection balance, the ratio $\sigma^{2}{ }_{m} / \sigma^{2}{ }_{G}$ is approximately equal to the average selection coefficient against heterozygous mutations and its reciprocal approximates the mean number of generations that a new mutation persists in a population before being removed by selection (Barton 1990; Kondrashov and Turelli 1992; Crow 1993a). Using this approach, Crow $(1993 a, b)$ has estimated that the mean persistence time of viability mutations in Drosophila is approximately 40 generations, both for mild detrimentals and recessive lethals. This result implies an average deleterious effect of about $2.5 \%$ in heterozygotes, which is very similar to the estimates obtained from the mutation-accumulation experiments of Mukai and Ohnishi. Nearly identical results have been obtained for mutations influencing life-history characters in Daphnia (Lynch et al. 1998). In a review of the data for a diversity of characters and species, all estimates of the mean persistence time $\left(\sigma^{2}{ }_{m} / \sigma^{2}{ }_{G}\right)$ were less than 1000 generations and more than half of them were less than 100 generations, with those for life-history characters averaging approximately 50 generations (Houle et al. 1996). These results are again broadly compatible with the existence of a common pool of deleterious mutations with heterozygous effects on the order of $0.1 \%$ to $1.0 \%$.

Wayne and Mackay (1998) recently estimated the persistence times for thorax length and ovariole number in D. melanogaster to be more than 10,000 generations, which would imply very low selection coefficients for mutant alleles. However, their estimates of $\sigma^{2}{ }_{m}$ were derived from inbred lines maintained at a population size of twenty flies/line, which allows the operation of substantial selection, and in turn, must result in upwardly biased estimates of persistence times.

\section{CONTRASTING RESUlts From BACTERIA, Worms, Plants, and Flies}

Although we view the classical interpretation of the results of Mukai and Ohnishi as not being far off the mark for flies, there also seems little question that real differences in the 
genomic deleterious mutation rate exist among different organisms. For example, a mutation-accumulation experiment performed on the bacterium Escherichia coli yielded results that were qualitatively very similar to those found by Mukai and Ohnishi-a pronounced decline in mean fitness and an equally pronounced increase in the variance among lines $(\mathrm{Ki}$ bota and Lynch 1996). However, the estimate of $U_{\min }$ obtained by the Bateman-Mukai method was only 0.00017 per cell division. The only other arthropod for which data are available, D. pulex, yields Bateman-Mukai estimates of $U_{\min }$ that are quite comparable to those for Drosophila, although those for $C$. elegans and A. thaliana are strikingly lower (Table 2).

Bacteria, worms, plants, and flies differ in many traits that could affect the genomic mutation rate. One obvious difference is the number of germline cell divisions per generation, which for D. melanogaster is about 36 (Drost and Lee 1995), and for C. elegans is eight to nine (Wilkins 1983). A second obvious difference is the activity of transposable elements in flies. As noted above, about $40 \%$ of all mutations in laboratory stocks of $D$. melanogaster appear to be a consequence of transposition events, whereas there is no detectable transposable element activity in the lines of E. coli and C. elegans that have been subjected to mutation-accumulation experiments. A third difference is the ploidy level of metazoans relative to $E$. coli. Taking these three factors into account, we can estimate the minimum deleterious mutation rate per haploid genome per cell division in $D$. melanogaster and $C$. elegans unassociated with transposable element activity: $(0.6$ $\times 0.6) /(2 \times 36)=0.005$ and $(0.05 \times 1.0) /(2 \times 8.5)=$ 0.003 , respectively. Although there is a nearly twofold difference in these estimates, this can hardly be regarded as significant given the crude nature of the analysis.

Aside from the fact that this scaling yields similar estimates of the minimum genomic deleterious mutation rate for species from two different phyla, the most striking aspect of these results is their qualitative consistency with recent estimates of the minimum total genomic mutation rate per cell division. Deriving estimates from observations at the molecular level, Drake (1991) concluded that the minimum total number of mutations is roughly constant in all DNA-based microbes, $\sim 0.003$ per haploid genome per cell division. More recent estimates for flies, worms, and mammals yielded an average estimate of approximately 0.006 per effective genome (coding DNA), which was not significantly different from the estimate for microbes (Drake et al. 1998). This estimate, which does not include transposition events, is only about $50 \%$ greater than our average minimum estimate of the haploid genomic deleterious mutation rate per cell division in worms and flies (0.004). There is a clear need for additional information of this kind on a diversity of organisms with well-known mutational properties, as approximate constancy of the genomic deleterious mutation rate per cell division would provide a powerful basis for indirectly inferring the mutational pressure operating on organisms with diverse modes of reproduction.

\section{UnAnswered Questions And Future Research}

In summary, a broad array of independent data on a diversity of organisms is compatible with the view that the genomic deleterious mutation rate is frequently on the order of 0.1 to 1.0 per individual per generation in multicellular eukaryotes and that the average homozygous and heterozygous effects of such mutations are typically less than $5 \%$. Although none of the empirical analyses that we have presented are entirely without problems, it seems unlikely that their qualitative agreement has arisen purely by chance. However, there seem to be legitimate reasons for concern that some of the conflicting conclusions obtained recently by distribution-based analyses may have resulted from violations of the assumptions of the models. The fact that E. coli, C. elegans, and $A$. thaliana appear to have lower genomic deleterious mutation rates than early estimates from Drosophila does not provide compelling evidence that the Drosophila results are flawed. Rather, there are plausible biological reasons for expecting the genomic mutation rates in these species to be lower than that in $D$. melanogaster. If this view is correct, then $U>0.6$ can be expected for some organisms with larger genome sizes and longer generation times than Drosophila, a point to which we will return below.

This being said, it is clear that there is still substantial need for additional work on the properties of spontaneous deleterious mutations. The validity of any such studies will continue to be subject to methodological scrutiny regarding the extent to which the control population remains evolutionarily stable. Because the magnitude of change observed in mutation-accumulation lines is often fairly small, even minor evolutionary changes in a control can lead to major interpretative difficulties. The maintenance of a large population as a control does not guarantee evolutionary stability. Indeed, the larger the control population, the more likely it is to accumulate rare beneficial mutations. For genetically variable base populations that have been moved from the field to a laboratory setting, there will generally be substantial opportunity for evolutionary adaptation to the novel environment, and even for populations that have long been adapted to laboratory life, there is still a possibility of fixation of new beneficial mutations, as well illustrated by the long-term evolution experiments of Lenski and colleagues (Lenski et al. 1991; Lenski and Travisano 1994). In addition, control populations kept in an active reproductive state sometimes run a risk of contamination by outside immigrants or by members of the parallel mutation-accumulation lines. The only way to eliminate these types of problems is to maintain the control in an evolutionarily inert state-seeds for plants or frozen embryos for animals. Even controls of this type are subject to the possibility that the storage process is mutagenic. However, provided the mutagenic properties of storage are not progressive, so that the performance of rejuvenated individuals remains constant in time, this is not a serious concern. When extended to experimental lines, the storage of individuals can also greatly enhance the statistical power of a mutation-accumulation experiment because individuals experiencing varying numbers of generations of mutation accumulation can be evaluated side by side in a common environment.

The Distribution of Mutational Effects.-Given the numerous assumptions underlying the distribution-based methods of analysis introduced by Keightley $(1994,1996)$ and García-Dorado (1997), it is clear that these methods need to 
be subjected to stringent sensitivity analyses before being widely adapted. A simple way to evaluate the power of distribution-based approaches would be to apply them to datasets such as those in Figure 1, where each genotype is known to contain exactly one mutation whose small effects on fitness are known to a fairly high degree of accuracy. A key issue is whether distance-based approaches will recover $U=1$ with such idealized datasets, under the otherwise usual assumptions of the models. To the extent that the technical issues of bias and sensitivity can be dealt with adequately, there will still be a clear need for new ML and MD algorithms that take into account multigenerational surveys. All current applications have been restricted to a single (usually, the final) assay, thereby ignoring a substantial amount of data. The computational demands of such methods will be very high, but recent progress in this area suggests that the increase in statistical power may be substantial (P. Keightley, pers. comm.).

Alternative means of empirically establishing the distribution of mutational effects are clearly desirable. One possible approach is to perform a set of mutation-accumulation experiments with lines of different effective sizes, while allowing for selection. Because the upper limit to the selection coefficient that is vulnerable to random genetic drift is proportional to $1 / N_{e}$, the smaller the effective size of a line, the larger the fraction of new mutations that will be impervious to selection. To our knowledge, only one experiment of this sort has been attempted. In addition to their standard protocol of taking fly lines through bottlenecks of single males, Mukai et al. (1972) maintained lines at a tenfold higher density. The absence of a density effect in these experiments provides further support for the existence of a common class of mutations with small deleterious effects. Further experiments of this nature with a properly designed control would provide a direct test of the hypothesis that small populations are subject to progressive mutational deterioration (Lynch et al. 1993, 1995a,b; Lande 1994; Schultz and Lynch 1997) and would help reveal the critical population size beyond which mutational degradation is unlikely, an issue of considerable importance to conservation biology.

By their very nature, mutations with small effects are difficult to study. However, as noted in Figure 1, microbial systems such as $E$. coli and $S$. cerevisiae provide powerful approaches to estimating the selection coefficients associated with mutations with small effects. By use of a microtiter plate reading system, highly accurate growth curves can be rapidly acquired for numerous replicates of large numbers of lines (Blanchard et al., unpubl.), and with appropriate markers, mutant lines can be competed directly against their nonmutant ancestors. Both approaches offer the opportunity to detect mutations with selection coefficients as small as 0.001 , and with an appropriate degree of replication, smaller than that.

A central issue that needs to be evaluated is the extent to which the directionality and distribution of mutational effects depends upon the premutational phenotype. This question lies at the heart of the two alternative evolutionary models that have been suggested for quantitative traits - the continuumof-alleles model of Kimura (1965), Lande (1975), and Lynch and Hill (1986) assumes that the effects of new mutations are symmetrically distributed around the effects of their an- cestral alleles, whereas the house-of-cards model of Bulmer (1972) and Turelli (1984) and the finite-alleles model of Zeng and Cockerham (1991) assume that the distribution of mutant alleles is independent of the premutational state. Under the former model, mutation does not constrain the possible range of phenotypic variation in a species, whereas under the latter model, extreme genotypes tend to mutate back toward the center of the range of variation. Given the central significance of these two alternative views of the spectrum of the quantitative effects of mutation, it is remarkable that there are essentially no data bearing on the matter. Insight could be acquired by performing mutation-accumulation experiments on genotypes that have been derived from natural populations with different mean phenotypes.

The Effect of Generation Length.-The qualitative analyses presented above suggest the possibility that the per generation rate of polygenic mutation scales at least crudely with generation time. If so, we might expect to see this pattern reflected in the mutational variance parameters $h^{2}{ }_{m}$ and/or $C V_{m}$. We tested this possibility by using the Houle et al. (1996) dataset on these parameters, supplemented with the more recent estimates (summarized for Drosophila in Table 1, and for other species, available on request). Applying analysis of covariance, with trait class as a classification variable, to the grouping 2 of Houle et al. (1996), where the median estimates for each trait type within species are analyzed, we find a significant correlation between generation time $(P<0.012)$ and $h^{2}{ }_{m}$ (Fig. 2). A very similar relationship arises regardless of whether all of the existing data are combined or whether the analysis is confined to life-history traits. Although the data are limited to only a few species and there is considerable scatter in the relationship, $h^{2}{ }_{m}$ does not appear to scale linearly with generation time, but with approximately the square root of the latter (the slope on a log scale being $0.45 \pm 0.17$ ). Arriving at a mechanistic interpretation for this pattern is not straightforward because mutational heritabilities are not just functions of the mutation rate, but of the average effects of mutations and of the environmental component of variance as well. Changes in both $\sigma^{2}{ }_{m}$ and $\sigma_{E}^{2}$ may be involved, as the average species-specific values of $C V_{m}$ are also positively associated with generation time (with regression slope 0.27 $\pm 0.13 ; P=0.036$ ), whereas $C V_{E}$ is negatively correlated with generation time, although not quite significantly $(P=$ $0.08)$.

It is possible that generation time is not a fundamental causal mechanism of the mutation rate, but is simply highly correlated with one or more variables that do directly influence the mutation rate. Roughly speaking, species with longer generation times can be expected to have greater numbers of germline cell divisions, so if mutations arise primarily during periods of cell division, this could explain in part the pattern in Figure 2. The idea that the genomic mutation rate per generation scales with the number of cell divisions is supported by the analyses of Drake et al. (1998), which suggest that the number of mutations per effective genome per cell division is approximately constant across a diversity of eukaryotic species, and by the observation that the human male mutation rate per generation increases with the age of reproduction (Crow 1993b). However, it is clear that cell division number per generation is not the sole determinant of the 

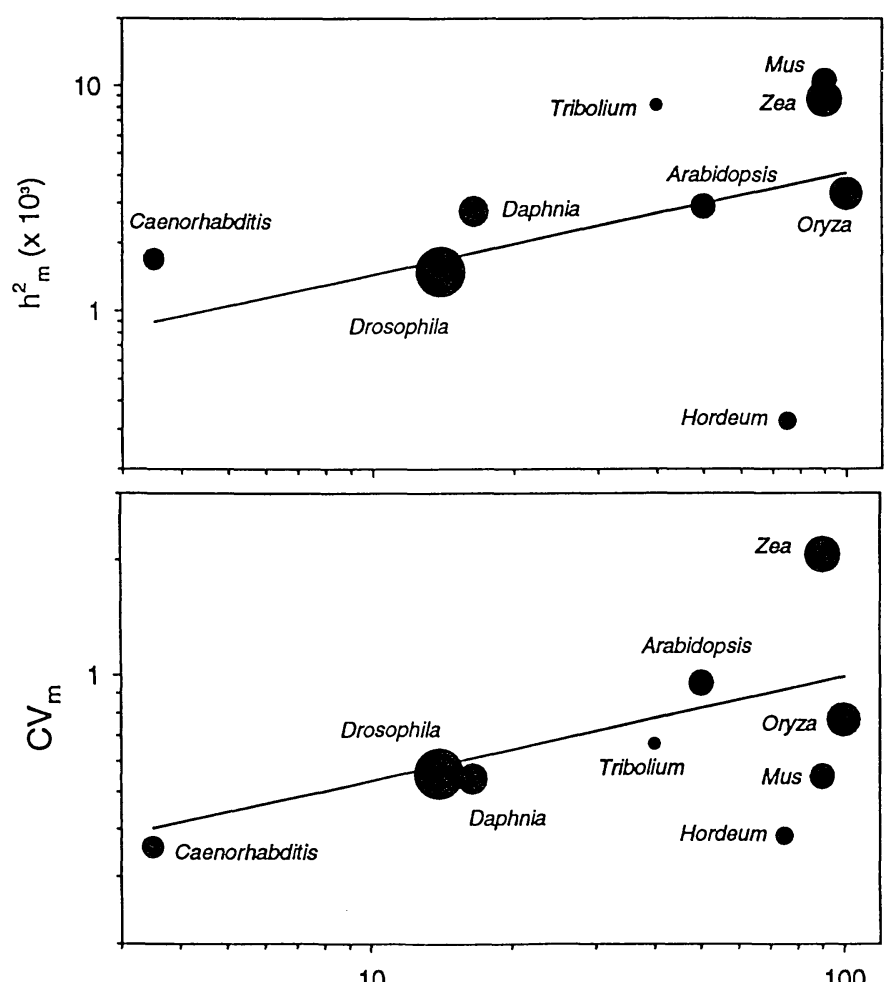

Generation time (days)

FIG. 2. The relationship between generation time and average mutational heritabilities and mutational CVs for species for which estimates are available. Data are taken from Table 1 and additional references cited in Houle et al. (1996) and Lynch and Walsh (1998). The centers of the circles are least-squares means from an ANCOVA, which takes into account the trait class. The areas of the circles are proportional to the number of traits studied in each species.

genomic mutation rate. For example, the mutation rate per locus in humans appears to be substantially less than that in mice and flies, on a per generation, per cell division, and per year basis (Drost and Lee 1995).

A simple way to test for a direct role of generation time in the determination of $U$ is to perform parallel mutationaccumulation experiments with an iteroparous organism, propagating different sets of lines at different parental ages. If the per generation mutation rate scales with absolute time, then after a fixed amount of time has elapsed, the total amounts of mutational divergence and decline should be approximately equivalent among all sets of lines regardless of their age of propagation. In addition, if the generation time hypothesis is qualitatively correct, then organisms with much shorter generation times than worms and Arabidopsis should exhibit lower estimates of $U_{\min }$ and perhaps lower estimates of $h^{2}{ }_{m}$. Yeast is an obvious candidate for such a study. The converse should hold for organisms with longer generation times, and given the potential implications of spontaneous deleterious mutation for our own species (below), a vertebrate model system such as the mouse or zebrafish would be logical candidates.

Environmental Dependence of Mutational Effects.-A central concern with the results of all mutation-accumulation experiments is the extent to which the results depend on the assay environment. Most such experiments employ benign conditions (optimal temperatures, unlimited resources, and low densities of individuals) during both the mutation-accumulation and assay phases. However, a number of recent studies with Drosophila have shown that fitness effects of spontaneous mutations are strongly environmentally dependent and have pronounced genotype $\times$ environment interactions (Kondrashov and Houle 1994; Fry et al. 1996, 1999; Fernández and López-Fanjul 1997; Wayne and Mackay 1998). None of these studies has revealed a class of mutations that is deleterious in one environment and beneficial in another, but the possibility remains that mutations that are deleterious in one environment may be effectively neutral in others.

In addition, it is uncertain whether it is primarily the number of expressed mutations or the distribution of their fitness effects that is altered in a harsher assay environment. In yeast ( $S$. cerevisiae), a substantial amount is known about the molecular response to extreme conditions, and it appears that the majority of genes are downregulated during stress response, while a minority of genes are induced by stress (Rüis and Schüller 1995). For example, heat shock reduces the levels of more than 300 of 500 proteins observed and leads to the induction of about 80 proteins (Miller et al. 1982). For well-characterized systems like this, it may be possible to achieve a mechanistic understanding of the environmental dependence of mutational parameters.

Elucidation of these issues is central to our understanding of the relevance of spontaneous deleterious mutation for the viability of populations. If the effects of individual mutations are magnified in harsher environments, then laboratory experiments performed in benign environments may exaggerate the population-level consequences of deleterious mutations because it is only mutations with relatively small effects that are likely to accumulate by random genetic drift. However, if the actual number of mutations that influence fitness is magnified in a harsh environment, with the distribution of effects remaining the same, then laboratory assays will typically underestimate the threat of mutations for wild populations.

Epistasis.-Finally, we note that the extent to which deleterious mutations act independently versus epistatically is a critical area for future research. The fate and populationlevel consequences of mutant alleles can depend strongly on their interactive effects. For example, when mutations have independent effects on fitness, the equilibrium mean fitness of an effectively infinite population is $e^{-U}$ (Kimura and Maruyama 1966; Crow 1970), but with synergistic epistasis (fitness declines at an accelerating rate with increasing numbers of mutation) the mean fitness of a sexual population increases above $e^{-U}$, whereas with diminishing-returns epistasis the opposite occurs (Charlesworth 1990). In contrast, the mean fitness of an infinite asexual population is independent of the form of epistasis. Under the belief that mutations are likely to interact synergistically, this has led to the suggestion that sexual reproduction may prevail in higher organisms because it results in the maintenance of a lower mutation load (Kondrashov 1982, 1988, 1994; Charlesworth 1990). However, the generality of this argument breaks down in finite popu- 
lations except in the unlikely situation in which mutations have constant single-locus effects (Lynch et al. 1993; Butcher 1995; Schultz and Lynch 1997). Because the magnitude of the selection coefficient that maximizes the rate of fitness loss in a finite population is approximately $1 /\left(2 N_{e}\right)$, the degree to which epistasis alters the vulnerability of a population to mutation depends on the extent to which cumulative epistasis modifies the density of mutations with selection coefficients in the vicinity of $1 /\left(2 N_{e}\right)$.

Although further theoretical work on these issues will continue to be illuminating, the issue of whether epistatic interactions even exist among mildly deleterious mutations will only be resolved by close empirical scrutiny. Most of the theoretical emphasis on synergistic epistasis was motivated by Mukai's (1969) demonstration of an accelerating decline of fitness with increasing numbers of mutations, despite the fact that this result was based on a plot of only seven nonindependent datapoints, and despite the fact that subsequent studies with the same species (Ohnishi 1977a,b) failed to make the same observation. A few other studies have led to the suggestion that epistatic interactions among deleterious mutations are synergistic (Malmberg 1977; Chao 1988; de Visser et al. 1996), but still other analyses have pointed to either a complete absence of epistasis or to the presence of a diversity of epistatic interactions (including synergistic and diminishing-returns) between various pairs of mutant alleles (de Visser et al. 1997a,b; Elena and Lenski 1997).

A significant limitation of many of these studies is the reliance on the temporal pattern of fitness decline in mutationaccumulation lines as an indicator of the cumulative pattern of epistatic effects. In principle, any such pattern can be biased by the operation of selection during the mutationaccumulation phase, by a change in mutation rate over the course of the experiment (due, e.g., to a change in metabolic activity) or by the saturation of mutational hotspots (such as microsatellite arrays with phenotypic effects). An increase in the rate of transposition with increasing copy number of transposable elements (Nuzhdin et al. 1996; Pasyukova et al. 1998) can result in a misleading appearance of synergistic epistasis. These kinds of interpretative difficulties can be avoided entirely by performing genetic analyses on crosses between mutant and control lines. With haploid lines, for example, greater similarity of the $F_{1}$ fitness to the control implies synergistic epistasis, whereas greater similarity to the mutant line implies diminishing-returns epistasis.

\section{Mutation Accumulation in Humans}

Nearly 50 years ago, Muller (1950) suggested that mildly deleterious mutations occur much more frequently than lethals, that the genomic mutation rate for humans is probably greatly in excess of that for flies, and that modern technological and medical innovations have encouraged the accumulation of mutations that otherwise would be purged in a harsher environment. He went on to warn that as a consequence of such mutational buildup, the time and effort necessary to counteract the effects of our mounting genetic afflictions is expected to increase. Crow (1993a,b, 1997) and Kondrashov (1995) have also called attention to the fact that mutations are almost certainly now accumulating in the hu- man population more rapidly than they are being eliminated. If our genomic mutation rate is increasing as a consequence of the physical and chemical alterations of the environment, as seems likely, then the situation is exacerbated.

Based on limited information, Muller (1950) surmised that the human mutation rate to deleterious alleles is on the order of 0.1 to 2.0 per genome. There is still a great deal of uncertainty over these estimates, but modern molecular data suggest that the actual number could be higher. As pointed out by Crow (1993b, 1997) and Kondrashov (1995), the total human mutation rate must be at least 100 per individual per generation. This can be easily seen by noting that: (1) the human diploid genome contains approximately $6.6 \times 10^{9}$ nucleotides, (2) the mutation rate per site is approximately $2.4 \times 10^{-9}$ per year, based on observed rates of nucleotide substitutions per synonymous site in protein-coding genes in mammals (this is a downwardly biased estimate because it does not take into account codon bias or other types of mutations such as insertions and deletions); and (3) the generation time for humans is approximately 20 years. Multiplying these three numbers together, we obtain a downwardly biased estimate of the genomic mutation rate of 317 . Much of the DNA in the human genome may be entirely dispensable, and arguments presented in Kondrashov (1995) suggest that this fraction may be as large as 90\%, whereas Drake et al. (1998) suggest a fraction of $97.5 \%$. Taking the latter as a conservative estimate, as well as the fact that about $85 \%$ of mutations in functional DNA in animals are subject to purifying selection (Lynch and Blanchard 1998), then the deleterious mutation rate in humans is suggested to be on the order of seven per genome per generation.

Another simple argument for the human genomic deleterious mutation rate being substantially in excess of that for invertebrates is the fact that the number of coding genes per genome is substantially inflated in vertebrates due to the two polyploidization events that preceded the divergence of the major vertebrate lineages (Lundin 1993; Holland et al. 1994) as well as to subsequent tandem duplications. On the order of $50 \%$ of all gene duplicates in mammals appear to have been preserved (Nadeau and Sankoff 1997), and they are not simply redundant in function, as most gene duplicates have evolved unique patterns of tissue-specific expression. Although the mutation rate per locus per generation in humans appears to be about $60 \%$ of that in Drosophila (Drost and Lee 1995), the mammalian genome contains about 4.5 times as many protein-coding genes. Thus, genome size considerations alone suggest that the genomic deleterious mutation rate in humans is probably at least double that in flies.

A third way to estimate the genomic deleterious mutation rate in humans is to adhere to the supposition of Drake et al. (1998) that the mutation rate per effective genome is approximately constant in metazoans on a per cell division basis. The results we cited above for Drosophila and Caenorhabditis suggest a minimum deleterious mutation rate per haploid genome per cell division of about 0.004 . Thus, because there are an average of approximately 200 germline cell divisions per generation in humans (Drost and Lee 1995), these estimates extrapolate to a minimum of about 1.6 deleterious mutations per human diploid genome per generation (ignoring the increase in genome size). 
As noted above, in the absence of selection, the asymptotic rate of accumulation of mutational load in a population is equal to the product of the genomic mutation rate and half the average homozygotic effect of a mutation. The numbers are very rough, but if we take $U=2$ and $E(a)=-0.02$ to be conservative estimates of these quantities, the potential per generation buildup of mutational damage in humans is approximately $4 \%$. The magnitude by which the realized damage will be less than $4 \%$ is a function of the efficiency of selection against new mutations. In societies living under more harsh conditions, deleterious mutation accumulation may be minimal, but nations with very modern medical practices may be asymptotically approaching the maximum rate. If this is the case, we may expect to see substantial decline in the general vigor of our population within a time frame of a dozen generations or so. Obvious candidate characters for monitoring include problems with visual acuity, tooth alignment, child-birth difficulties, and infertility, all of which historically must have been subject to strong selection, but which are now greatly modified by medical intervention.

We conclude that Muller's (1950) concern with mutational buildup in the human population is legitimate. A generation ago, the major threat to the survival of our species appeared to be nuclear war and today we are preoccupied with the potential consequences of global warming and other aspects of environmental deterioration. There is some hope that environmental problems can be ameliorated by technological change, but the social and practical obstacles to reducing our load of mutations are enormous. Even if society eventually adopts widespread policies of genetic counseling and/or modification, such procedures hardly seem applicable to our most common pool of mutations - those with very minor individual effects. Improved medical and technological procedures may transform the selective landscape such that the negative consequences of deleterious mutations are reduced to near insignificance, but the price of increased societal investment in such procedures may not be trivial.

\section{ACKNOWLEDGMENTS}

This work was supported by National Institute of Health grant RO1-GM36827 and National Science Foundation grant DEB 96-29775 to ML. We thank R. Frankham, J. Gillespie, A. Kondrashov, and R. Lande for helpful comments.

\section{Literature Cited}

AKASHI, H. 1995. Inferring weak selection from patterns of polymorphism and divergence at "silent" sites in Drosophila DNA. Genetics 139:1067-1076.

Ayala, F. J. 1968. Evolution of fitness. II. Correlated effects of natural selection on the productivity and size of experimental populations of Drosophila serrata. Evolution 22:55-65.

BAILEY, D. W. 1959. Rates of subline divergence in highly inbred strains of mice. J. Heredity 50:26-30.

BARTON, N. H. 1990. Pleiotropic models of quantitative variation. Genetics 124:773-782.

BATEMAN, A. J. 1959. The viability of near-normal irradiated chromosomes. Int. J. Rad. Biol. 1:170-180.

Bauer, V. L., AND C. F. Aquadro. 1997. Rates of DNA sequence evolution are not sex-biased in Drosophila melanogaster and D. simulans. Mol. Biol. Evol. 14:1252-1257.

Bulmer, M. G. 1972. The genetic variability of polygenic char- acters under optimizing selection, mutation, and drift. Genet. Res. 19:17-25.

1991. The selection-mutation-drift theory of synonymous codon usage. Genetics 129:897-907.

Butcher, D. 1995. Muller's ratchet, epistasis and mutation effects. Genetics 141:431-437.

ChaO, L. 1988. Evolution of sex in RNA viruses. J. Theor. Biol. 133:99-112.

Charlesworth, B. 1990. Mutation-selection balance and the evolutionary advantage of sex and recombination. Genet. Res. 55: 199-221.

1991. The evolution of sex chromosomes. Science 251: $1030-1033$.

Charlesworth, B., and K. A. Hughes. 1996. Age-specific inbreeding depression and components of genetic variance in relation to the evolution of senescence. Proc. Natl. Acad. Sci. USA 93:6140-6145.

Charlesworth, B., And K. A. Hughes. In press. The maintenance of genetic variation in life history traits. In R. S. Singh and C. B. Krimbas, eds. Evolutionary genetics from molecules to morphology. Cambridge Univ. Press, Cambridge, U.K.

Charlesworth, B., D. Charlesworth, and M. T. Morgan. 1990 Genetic loads and estimates of mutation rates in highly inbred plant populations. Nature 347:380-382.

Charlesworth, B., A. Lapid, and D. Canada. 1992. The distribution of transposable elements within and between chromosomes in a population of Drosophila melanogaster. I. Element frequencies and distribution. Genet. Res. 60:103-114.

Charlesworth, B., M. T. Morgan, and D. Charlesworth. 1993 The effect of deleterious mutations on neutral molecular evolution. Genetics 134:1289-1303.

Charlesworth, D., and B. Charlesworth. 1987. Inbreeding depression and its evolutionary consequences. Annu. Rev. Ecol. Syst. 18:237-268.

Charlesworth, D., M. T. Morgan, and B. Charlesworth. 1992. The effect of linkage and population size on inbreeding depression due to mutational load. Genet. Res. 59:49-61.

Charlesworth, D., E. E. Lyons, And L. B. Litchfield. 1994 Inbreeding depression in two highly inbreeding populations of Leavenworthia. Proc. R. Soc. Lond. B Biol. Sci. 258:209-214.

Clark, A. G., L. Wang, and T. Hulleberg. 1995. Spontaneous mutation rate of modifiers of metabolism in Drosophila. Genetics 139:767-779.

Crow, J. F. 1970. Genetic loads and the cost of natural selection, Pp. 128-177 in K. Kojima, ed. Mathematical topics in population genetics. Springer-Verlag, Berlin.

. 1993a. Mutation, mean fitness, and genetic load. Oxford Surv. Evol. Biol. 9:3-42

- 1993b. How much do we know about spontaneous human mutation rates? Environ. Mol. Mutagen. 21:122-129.

1997. The high spontaneous mutation rate: is it a health risk? Proc. Natl. Acad. USA 94:8380-8386.

Crow, J. F., AND M. J. Simmons. 1983. The mutation load in Drosophila. Pp. 1-35 in M. Ashburner, H. L. Carson, and J. N. Thompson, Jr., eds. The genetics and biology of Drosophila. Vol. 3c. Academic Press, New York.

DENG, H.-W. 1998. Estimation of deleterious-mutation rate and effects in outcrossing populations. Genetics 150:945-956.

DENG, H.-W., AND M. LYNCH. 1996. Estimation of deleterious. mutation parameters in natural populations. Genetics 144:349360 .

- 1997. Inbreeding depression and inferred deleterious mutation parameters in Daphnia. Genetics 147:147-155.

DE Visser, J. A. G. M., R. F. Hoekstra, and H. van den Ende. 1996. The effect of sex and deleterious mutations in Chlamydomonas. Proc. R. Soc. Lond. B Biol. Sci. 263:193-200.

- 1997a. An experimental test for synergistic epistasis and its application in Chlamydomonas. Genetics 145:815-819.

- $1997 \mathrm{~b}$. Test of interaction between genetic markers that affect fitness in Aspergillus niger. Evolution 51:1499-1505.

Dobzhansky, T., H. Levene, B. Spassky, and N. Spassky. 1959. Release of genetic variability through recombination. III. Drosophila prosaltans. Genetics 44:75-92. 
DraKe, J. W. 1991. A constant rate of spontaneous mutation in DNA-based microbes. Proc. Natl. Acad. Sci. USA 88:71607164.

Drake, J., B. Charlesworth, D. Charlesworth, and J. F. Crow. 1998. Rates of spontaneous mutation. Genetics 148:1667-1686.

Drost, J. B., AND W. R. LEE. 1995. Biological basis of germline mutation: comparisons of spontaneous germline mutation rates among Drosophila, mouse, and human. Environ. Mol. Mut. 25, Suppl. 26:48-64.

Elena, S. F., AND R. E. Lenski. 1997. Test of synergistic interactions among deleterious mutations in bacteria. Nature 390: 395-398

Elena, S. F., L. Ekunwe, N. Hajela, S. A. Oden, and R. E. Lenski. 1998. Distributions of fitness effects caused by random insertion mutations in Escherichia coli. Genetica 102/103:349-358.

FERnÁNDEZ, J., ANd C. LóPEZ-FAnJul. 1996. Spontaneous mutational variances and covariances for fitness-related traits in Drosophila melanogaster. Genetics 143:829-837.

-1997. Spontaneous mutational genotype-environment interaction for fitness-related traits in Drosophila melanogaster. Evolution 51:856-864.

FEsting, M. F. W. 1973. A multivariate analysis of subline divergence in the shape of mandible in C57BL/Gr mice. Genet. Res. 21:121-132.

Force, A., M. Lynch, B. Pickett, A. Amores, Y.-L. Yan, and J. Postlethwait. 1999. Preservation of duplicate genes by complementary, degenerative mutations. Genetics 151:1531-1545.

FRANKHAM, R., AND D. A. LOEBEL. 1992. Modeling conservation genetics problems using captive Drosophila populations: rapid genetic adaptation to captivity. Zoo Biol. 11:333-342.

Fry, J. D., K. A. DeRonde, And T. F. C. Mackay. 1995. Polygenic mutation in Drosophila melanogaster: genetic analysis of selection lines. Genetics 139:1293-1307.

Fry, J. D., S. L. Heinsohn, and T. F. C. Mackay. 1996. The contribution of new mutations to genotype-environment interaction for fitness in Drosophila melanogaster. Evolution 50: 2316-2327.

. 1998. Heterosis for viability, fecundity, and male fertility in Drosophila melanogaster: comparison of mutational and standing variation. Genetics 148:1171-1188.

Fry, J. D., P. D. Keightley, S. L. Heinsohn, and S. V. Nuzhdin. 1999. New estimates of the rates and effects of mildly deleterious mutation in Drosophila melanogaster. Proc. Natl. Acad. Sci. USA 96:574-579.

García-Dorado, A. 1997. The rate and effects distribution of viability mutation in Drosophila: minimum distance estimation. Evolution 51:1130-1139.

García-Dorado, A., And J. M. Marín. 1998. Minimum distance estimation of mutational parameters for quantitative traits. Biometrics 54:214-230.

García-Dorado, A., J. L. Monedero, and C. López-Fanjul. 1998. The mutation rate and the distribution of mutational effects of viability and fitness in Drosophila melanogaster. Genetica 102/103:255-265.

Gilligan, D. M., L. M. Woodworth, M. E. Montgomery, D. A. BRISCOE, AND R. FRANKHAM. 1997. Is mutation accumulation a threat to the survival of endangered populations? Cons. Biol. $11: 12351-1241$

Grewal, M. S. 1962. The rate of genetic divergence of sublines in the C57BL strain of mice. Genet. Res. 3:226-237.

Haldane, J. B. S. 1937. The effect of variation on fitness. Am. Nat. 71:337-349.

Hamilton, W. D. 1966. The moulding of senescence by natural selection. J. Theor. Biol. 12:12-45.

HoI-SEN, Y. 1972. Is subline differentiation a continuing process in inbred strains of mice? Genet. Res. 19:53-59.

Holland, P. W. H., J. García-Fernández, N. A. Williams, and A. Sidow. 1994. Gene duplications and the origins of vertebrate development. Devel., Suppl. 125-133.

Houle, D. 1992. Comparing evolvability and variability of quantitative traits. Genetics 130:195-204.

1998. How should we explain variation in the genetic variance of traits? Genetica 102/103:241-253.
Houle, D., D. K. Hoffmaster, S. Assimacopoulus, and B. Charlesworth. 1992. The genomic mutation rate for fitness in Drosophila. Nature 359:58-60.

Houle, D., K. A. Hughes, D. K. Hoffmaster, J. Ihara, S. Assimacopoulos, D. Canada, and B. Charlesworth. 1994a. The effects of spontaneous mutation on quantitative traits. I. Variance and covariance of life history traits. Genetics 138:773-785.

Houle, D., D. K. Hoffmaster, S. Assimacopoulus, and B. CHARLESWORTH. 1994b. Correction: the genomic mutation rate for fitness in Drosophila. Nature 371:358.

Houle, D., B. Morikawa, ANd M. LynCH. 1996. Comparing mutational heritabilities. Genetics 143:1467-1483.

Houle, D., K. A. Hughes, S. Assimacopoulos, and B. CharlesWORTH. 1997a. The effects of spontaneous mutation on quantitative traits. II. Dominance of mutations with effects on lifehistory traits. Genet. Res. 70:27-34.

Houle, D., A. S. Kondrashov, L. Y. Yampolsky, S. Caldwell, AND P. L. STEPONKUS. 1997b. The effect of cryopreservation on the lethal mutation rate in Drosophila melanogaster. Genet. Res. 3:209-213.

Hudson, R. R., AND N. L. Kaplan. 1995. Deleterious background selection with recombination. Genetics 141:1605-1617.

Johnston, M. O., And D. J. Schoen. 1995. Mutation rates and dominance levels of genes affecting total fitness in two angiosperm species. Science 267:226-229.

Kawecki, T. J., N. H. Barton, And J. D. Fry. 1997. Mutational collapse of fitness in marginal habitats and the evolution of ecological specialization. J. Evol. Biol. 10:407-429.

KeightLey, P. D. 1994. The distribution of mutation effects on viability in Drosophila melanogaster. Genetics 138:1315-1322. 1996. Nature of deleterious mutation load in Drosophila. Genetics 144:1993-1999.

1998. Inference of genome wide mutation rates and distributions of mutation effects for fitness traits: a simulation study. Genetics 150:1283-1293.

Keightley, P. D., and A. Caballero. 1997. Genomic mutation rates for lifetime reproductive output and lifespan in Caenorhabditis elegans. Proc. Natl. Acad. Sci. USA 94:3823-3827.

Keightley, P. D., AND O. OHNishi. 1998. EMS-induced polygenic mutation rates for nine quantitative characters in Drosophila melanogaster. Genetics 148:753-766.

Keightley, P. D., T. F. C. Mackay, and A. Caballero. 1993. Accounting for bias in estimates of the rate of polygenic mutation. Proc. R. Soc. Lond. B Biol. Sci. 253:291-296.

Keightley, P. D., A. Caballero, and A. García-Dorado. 1998. Surviving under mutation pressure. Curr. Biol. 8:R235-R237.

Kibota, T., AND M. LyNCH. 1996. Estimate of the genomic mutation rate deleterious to overall fitness in Escherichia coli. Nature 381:694-696.

KIMURA, M. 1965. A stochastic model concerning the maintenance of genetic variability in quantitative characters. Proc. Natl. Acad. Sci. USA 54:731-736.

1983. The neutral theory of molecular evolution. Cambridge Univ. Press, Cambridge, U.K.

Kimura, M., AND J. F. CROW. 1963. On the maximum avoidance of inbreeding. Genet. Res. 4:399-415.

Kimura, M., AND T. MARUyama. 1966. The mutational load with epistatic gene interactions in fitness. Genetics 54:1337-1351.

Kimura, M., AND T. OHTA. 1971. On the rate of molecular evolution. J. Mol. Evol. 1:1-17.

KondRAHSOV, A. S. 1982. Selection against harmful mutations in large sexual and asexual populations. Genet. Res. 40:325-332. - 1988. Deleterious mutations and the evolution of sexual reproduction. Nature 336:435-440.

. 1994. Muller's ratchet under epistatic selection. Genetics 136:1469-1473.

- 1995. Contamination of the genome by very slightly deleterious mutations: why have we not died 100 times over? J. Theor. Biol. 175:583-594.

Kondrashov, A. S., AND J. F. CROw. 1991. Haploidy or diploidy: which is better? Nature 351:314-315.

Kondrashov, A. S., AND D. Houle. 1994. Genotype-environment interactions and the estimation of the genomic mutation rate in 
Drosophila melanogaster. Proc. R. Soc. Lond. B Biol. Sci. 258: 221-227.

Kondrashov, A. S., AND M. Turelli. 1992. Deleterious mutations, apparent stabilizing selection and the maintenance of quantitative variation. Genetics 132:603-618.

KRIMBAS, C. B. 1961. Release of genetic variability through recombination. VI. Drosophila willistoni. Genetics 46:1323-1334.

LANDE, R. 1975. The maintenance of genetic variation by mutation in a polygenic character with linked loci. Genet. Res. 26:221235.

\section{mutations. Evolution 48:1460-1469.}

LANDE, R., AND D. W. Schemske. 1985. The evolution of selffertilization and inbreeding depression in plants. I. Genetic models. Evolution 39:24-40.

Langley, C. H., R. A. Voelker, A. J. L. Brown, S. Ohnishi, B. Dickson, AND E. Montgomery. 1981. Null allele frequencies at allozyme loci in natural populations of Drosophila melanogaster. Genetics 99:151-156.

LATTER, B. D. H. 1998. Mutant alleles of small effect are primarily responsible for the loss of fitness with slow inbreeding in Drosophila melanogaster. Genetics 148:1143-1158.

LatTer, B. D. H., AND J. C. Mulley. 1995. Genetic adaptation to captivity and inbreeding depression in small laboratory populations of Drosophila melanogaster. Genetics 139:255-266.

Lenski, R. E., AND M. Travisano. 1994. Dynamics of adaptation and diversification: a 10,000-generation experiment with bacterial populations. Proc. Natl. Acad. Sci. USA 91:6808-6814.

Lenski, R. E., M. R. Rose, S. C. Simpson, And S. C. TADler. 1991. Long-term experimental evolution in Escherichia coli. I. Adaptation and divergence during 2000 generations. Am. Nat. 138: $1315-1341$

LI, W.-H. 1980. Rate of gene silencing at duplicate loci: a theoretical study and interpretation of data from tetraploid fishes. Genetics 95:237-258.

LUNDIN, L. 1993. Evolution of the vertebrate genome as reflected in paralogous chromosomal regions in man and the house mouse. Genomics 16:1-9.

Lyman, R. F., F. Lawrence, S. V. Nuzhdin, and T. F. C. Mackay. 1996. Effects of single $P$ element insertions on bristle number and viability in Drosophila melanogaster. Genetics 143:277292.

LYNCH, M. 1988. The rate of polygenic mutation. Genet. Res. 51: $137-148$.

1994. The neutral theory of phenotypic evolution. Pp. 86108 in L. Real, ed. Ecological genetics. Princeton Univ. Press, Princeton, NJ.

- 1997. Mutation accumulation in nuclear, organelle, and prokaryotic transfer RNA genes. Mol. Biol. Evol. 14:914-925.

LYNCH, M., AND J. BLANCHARD. 1998. Deleterious mutation accumulation in organelle genomes. Genetica 102/103:29-39.

LyNCH, M., AND H.-W. DenG. 1994. Genetic slippage in response to sex. Am. Nat. 144:242-261.

LyNCH, M., AND W. G. HILl. 1986. Phenotypic evolution and neutral mutation. Evolution 40:915-935.

LyNCH, M., AND B. WALSH. 1998. Genetics and analysis of quantitative traits. Sinauer, Sunderland, MA.

Lynch, M., R. Burger, D. Butcher, And W. Gabriel. 1993. The mutational meltdown in asexual populations. J. Heredity 84: 339-344.

LynCH, M., J. CONERY, AND R. Burger. 1995a. Mutational meltdowns in sexual populations. Evolution 49:1067-1080.

. 1995b. Mutation accumulation and the extinction of small populations. Am. Nat. 146:489-518.

Lynch, M., L. Latta, J. Hicks, and M. Giorgianni. 1998. Mutation, selection, and the maintenance of life-history variation in natural population. Evolution 52:727-733.

MACKAY, T. F. C., R. F. LyMAN, AND M. S. JACKSON. 1992. Effects of $\mathrm{P}$ element insertion on quantitative traits in Drosophila melanogaster. Genetics 130:315-332.

Mackay, T. F. C., J. D. Fry, R. F. Lyman, and S. V. Nuzhdin. 1994. Polygenic mutation in Drosophila melanogaster: estimates from response to selection of inbred strains. Genetics 136:937951.

Mackay, T. F. C., R. F. Lyman, And W. G. Hill. 1995. Polygenic mutation in Drosophila melanogaster: non-linear divergence among unselected strains. Genetics 139:849-859.

MalmberG, R. L. 1977. The evolution of epistasis and the advantage of recombination in populations of bacteriophage T4. Genetics 86:607-621.

Martorell, C., M. A. Toro, and C. Gallego. 1998. Spontaneous mutation for life-history traits in Drosophila melanogaster. Genetica 102/103:315-324.

Miller, M. J., N. H. XuONG, ANd E. P. Guidushek. 1982. Quantitative analysis of the heat shock response of Saccharomyces cerevisiae. J. Bact. 151:311-327.

Morton, N. E., J. F. Crow, And H. J. Muller. 1956. An estimate of the mutational damage in man from data on consanguineous matings. Proc. Natl. Acad. Sci. USA 42:855-863.

MuKAI, T. 1964. The genetic structure of natural populations of Drosophila melanogaster. I. Spontaneous mutation rate of polygenes controlling viability. Genetics 50:1-19.

. 1969. The genetic structure of natural populations of Drosophila melanogaster. VII. Synergistic interaction of spontaneous mutant polygenes controlling viability. Genetics 61:749761.

- 1979. Polygenic mutation. Pp. 177-196 in J. N. Thompson, Jr. and J. M. Thoday, eds. Quantitative genetic variation. Academic Press, New York.

. 1980. The genetic structure of natural populations of Drosophila melanogaster. XIV. Effects of the incomplete dominance of the IN(2LR)SM1 ( $C y$ ) chromosome on the estimates of various genetic parameters. Genetics 94:749-761.

Mukai, T., And O. Yamaguchi. 1974. The genetic structure of natural populations of Drosophila. XI. Genetic variability in a local population. Genetics 82:63-82.

MUKAI, T., AND T. YAMAZAKI. 1968. The genetic structure of natural populations of Drosophila melanogaster. V. Coupling-repulsion effect of spontaneous mutant polygenes controlling viability. Genetics 59:513-535.

- 1971. The genetic structure of natural populations of Drosophila melanogaster. X. Developmental time and viability. Genetics 69:385-398.

Mukai, T., S. I. Chigusa, L. E. Mettler, and J. F. Crow. 1972. Mutation rate and dominance of genes affecting viability in Drosophila melanogaster. Genetics 72:335-355.

Muller, H. J. 1950. Our load of mutations. Am. J. Hum. Gen. 2 : $111-176$

Nadeau, J. H., And D. SAnKoff. 1997. Comparable rates of gene loss and functional divergence after genome duplications early in vertebrate evolution. Genetics 147:1259-1266.

NuZhdin, S. V., AND T. F. C. MACKAY. 1995. The genomic rate of transposable element movement in Drosophila melanogaster. Mol. Biol. Evol. 12:180-181.

Nuzhdin, S. V., J. D. Fry, and T. F. C. Mackay. 1995. Polygenic mutation in Drosophila melanogaster: the causal relationship of bristle number to fitness. Genetics 139:861-872.

Nuzhdin, S. V., E. G. Pasyukova, and T. F. C. MaCKay. 1996 Positive association between copia transposition rate and copy number in Drosophila melanogaster. Proc. R. Soc. Lond. B Biol. Sci. 263:823-831.

OHNishi, O. 1974. Spontaneous and ethyl methanesulfonate-induced mutations controlling viability in Drosophila melanogaster. Ph.D. diss., University of Wisconsin, Madison, WI

- 1977a. Spontaneous and ethyl methane-sulfonate induced mutations controlling viability in Drosophila melanogaster. I. Recessive lethal mutations. Genetics 87:519-527.

1977b. Spontaneous and ethyl methane-sulfonate induced mutations controlling viability in Drosophila melanogaster. II. Homozygous effect of polygenic mutations. Genetics 87:529545

1977c. Spontaneous and ethyl methane-sulfonate induced mutations controlling viability in Drosophila melanogaster. III. Heterozygous effect of polygenic mutations. Genetics 87:547556 
OHtA, T. 1992. The nearly netural theory of molecular evolution. Annu. Rev. Ecol. Syst. 23:263-286.

OKa, H. I., J. Hayashi, AND I. Shiojiri. 1958. Induced mutations of polygenes for quantitative characters in rice. J. Heredity 49: 11-14.

Pamilo, P., M. NeI, AND W.-H. LI. 1987. Accumulation of mutations in sexual and asexual populations. Genet. Res. 49:135146.

Partridge, L., AND N. H. Barton. 1993. Optimality, mutation and the evolution of aging. Nature 362:305-311.

Pasyukova, E. G., S. V. Nuzhdin, and D. A. Filatov. 1998. The relationship between the rate of transposition and transposable element copy number for copia and Doc retroposons of Drosophila melanogaster. Genet. Res. 72:1-11.

Perrot, V., S. Richerd, AND M. Valero. 1991. Transition from haploidy to diploidy. Nature 351:315-317.

Pletcher, S. D., D. Houle, And J. W. Curtsinger. 1998. Agespecific properties of spontaneous mutations affecting mortality in Drosophila melanogaster. Genetics 148:287-303.

RICE, W. R. 1994. Degeneration of a nonrecombining chromosome. Science 263:230-232.

Russell, W. A., G. F. Sprague, and L. H. Penny. 1963. Mutations affecting quantitative characters in long-term inbred lines of maize. Crop Sci. 3:175-178.

SAKAI, K.-I., AND A. SUZUKI. 1964. Induced mutation and pleiotropy of genes responsible for quantitative characters in rice. Rad. Bot. 4:141-151.

Santiago, E., J. Albornoz, A. Dominguez, M. A. Toro, and C. LÓPEZ-FANJUL. 1992. The distribution of spontaneous mutations on quantitative traits and fitness in Drosophila melanogaster. Genetics 132:771-781.

Schultz, S. T., AND M. Lynch. 1997. Deleterious mutation and extinction: effects of variable mutational effects, synergistic epistasis, beneficial mutations, and degree of outcrossing. Evolution 51:1363-1371.

Shabalina, S. A., L. Y. Yampolsky, and A. S. Kondrashov. 1997. Rapid decline of fitness in panmictic populations of Drosophila melanogaster maintained under relaxed natural selection. Proc. Natl. Acad. Sci. USA 94:13034-13039.

Sharp, P. M., AND W.-H. Li. 1989. On the rate of DNA sequence evolution in Drosophila. J. Mol. Evol. 28:398-402.
Simmons, M. J., AND J. F. Crow. 1977. Mutations affecting fitness in Drosophila populations. Annu. Rev. Genet. 11:49-78.

Spassky, B., N. Spassky, H. Levene, and T. Dobzhansky. 1958. Release of genetic variability through recombination. I. Drosophila pseudoobscura. Genetics 43:844-867.

SpIEss, E. B. 1959. Release of genetic variability through recombination. II. Drosophila persimilis. Genetics 44:43-58.

SpIEss, E. B., AND A. C. AlLEN. 1961. Release of genetic variability through recombination. VII. Second and third chromosomes of Drosophila melanogaster. Genetics 46:1531-1553.

Sprague, G. F., W. A. Russell, and L. H. Penny. 1960. Mutations affecting quantitative traits in the selfed progeny of double monoploid maize stocks. Genetics 45:855-866.

Thatcher, J. W., J. M. Shaw, and W. J. Dickinson. 1998. Marginal fitness contributions of nonessential genes in yeast. Proc. Natl. Acad. Sci. USA 95:253-257.

Turelli, M. 1984. Heritable genetic variation via mutation-selection balance: Lerch's zeta meets the abdominal bristle. Theor. Popul. Biol. 25:138-193.

VASSILIEVA, L. L., AND M. LYNCH. 1999. The rate of spontaneous mutation for life-history traits in Caenorhabditis elegans. Genetics 151:119-129.

WALLACE, B. 1956. Studies of irradiated populations of Drosophila melanogaster. J. Genet. 56:280-293.

WALSH, J. B. 1995. How often do duplicated genes evolve new functions? Genetics 110:345-364.

WAYNe, M. L., AND T. F. C. MACKAY. 1998. Quantitative genetics of ovariole number in Drosophila melanogaster. II. Mutational variation and genotype-environment interaction. Genetics 148: 201-210.

Weber, K. E., AND L. T. Diggins. 1990. Increased selection response in larger populations. II. Selection for ethanol vapor resistance in Drosophila melanogaster at two population sizes. Genetics 125:585-597.

WILKINS, A. S. 1992. Genetic analysis of animal development. 2d ed. Wiley-Liss, New York.

Zeng, Z.-B., AND C. C. Cockerham. 1991. Variance of neutral genetic variances within and between populations for a quantitative character. Genetics 129:535-553.

Corresponding Editor: T. Markow 